\title{
Winfried Thaa \\ Kulturkritik und Demokratie bei Max Weber und Hannah Arendt
}

\author{
»Alle ökonomischen Wetterzeichen \\ weisen nach der Richtung \\ zunehmender $>$ Unfreiheit « 1 .
}

\begin{abstract}
1. Einleitung
Die letzten zehn, zwölf Jahre sahen den Aufstieg Hannah Arendts zu einer nahezu unangreifbaren moralischen und politischen Autorität. Einen nicht geringen Anteil daran hatten Autoren der ehemaligen Neuen Linken, die Arendt bis weit in die achtziger Jahre hinein noch als Vertreterin eines »normativ-ontologischen Ansatzes « unter Konservatismusverdacht gestellt hatten ${ }^{2}$. Im Zentrum dieser Neuentdeckung des Arendtschen Denkens steht ihr »enthusiastischer Begriff des Politischen $\aleph^{3}$, der zum einen Anschlussmöglichkeiten für eine Konzeptualisierung der Neuen Sozialen Bewegungen bot, darüber hinaus aber auch grundsätzlicher verspricht, den gesellschaftskritischen Kern des Praxisbegriffes aus den Trümmern des Marxismus zu retten.

Bemerkenswert scheint mir dabei, dass die kultur- und modernitätskritischen $\mathrm{Pa}$ rallelen zwischen dem Denken Hannah Arendts auf der einen und dem von Max Webers Rationalisierungstheorie geprägten Denken der Frankfurter Schule auf der anderen Seite in der neueren Arendt-Diskussion kaum eine Rolle spielen. Schließlich war ja nicht nur die immense intellektuelle Wirkung der Frankfurter Schule während der sechziger und siebziger Jahre aufs engste mit der Kritik instrumenteller Vernunft (Horkheimer) bzw. technologischer Rationalität (Marcuse) verbunden. Auch die politisch einflussreichen Bewegungen der siebziger und achtziger Jahre zeichneten sich durch Fortschrittsskepsis und Modernitätskritik aus. Wo die neuere Diskussion explizit auf Arendts Kritik der Moderne eingeht, gilt ihr dies als höchst
\end{abstract}

1 Max Weber: "Zur Lage der bürgerlichen Demokratie in Russland « in: ders., Gesammelte Politische Schriften, Tübingen 1988 (1921), S. 63.

2 Mit entscheidend hierfür war die Ablehnung der Totalitarismustheorie durch die große Mehrheit der linken Intellektuellen in Deutschland. Zu weiteren Gründen der verspätet einsetzenden Arendt-Rezeption vgl. Michael Greven, »Hannah Arendt - Pluralität und die Gründung der Freiheit« in: Peter Kemper (Hg.), Die Zukunft des Politischen, Frankfurt a.M. 1993, S. 97-123.

3 Ernst Vollrath, »Hannah Arendt « in: Karl Graf Ballestrem / Henning Ottmann (Hg.), Politische Philosophie des 20. Jahrhunderts, München 1990, S. 18. 
bedenklicher, auf den Einfluss Heideggers zurückgehender Aspekt ihres Denkens, der dann in der Regel zugunsten einer diskurstheoretischen Lesart ihres Werkes relativiert wird ${ }^{4}$.

Der dominierende Einfluss des Habermasschen Verdikts gegen die »hemmungslose Vernunftskepsis « ${ }^{5}$ der ersten Generation der Frankfurter mag dazu beigetragen haben, das Motiv der Modernitäts- und Kulturkritik in den Hintergrund zu drängen, bzw. in ihm vor allem eine hochproblematische, auf den antiwestlichen deutschen Sonderweg verweisende Gemeinsamkeit zwischen den unterschiedlichsten Denkern des deutschen Sprachraums zu sehen. So nachvollziehbar das jüngste Interesse an Arendts Politikbegriff ist, so wenig kann jedoch ein Zweifel daran bestehen, dass die Kritik der Moderne nicht am Rand, sondern im Zentrum ihres Denkens steht. Mehr noch, der so ungemein populär gewordene Politikbegriff Arendts und ihre aus der amerikanischen Geschichte gewonnene Konzeption einer republikanischen Demokratie lassen sich überhaupt nur verstehen als Antwort auf die von ihr erfahrene Totalisierung von Herrschaft unter Bedingungen der Moderne. Während die konservative Kulturkritik in den ersten Jahrzehnten des 20. Jahrhunderts mit ihrer Gegenüberstellung von wahrer, an höheren Werten orientierter (und selbstverständlich deutscher) Kultur auf der einen und verflachter, utilitaristischer und amoralischer westlicher Zivilisation auf der anderen Seite antipolitisch orientiert war und in der Demokratie ein Symptom der Dekadenz und der naturwidrigen Nivellierung sah ${ }^{6}$, entfaltet Arendt ihren enthusiastischen Politikbegriff als Gegenkraft zu den freiheitsbedrohenden Tendenzen der Moderne.

Darin liegt eine interessante Parallele zum politischen Denken Max Webers. Auch Webers Auseinandersetzung mit der Moderne ist kulturkritisch geprägt, wie die Bücher von Wilhelm Hennis und Lawrence Scaff, lange zuvor aber auch schon

4 Beispielhaft hierfür ist das Arendt-Buch von Seyla Benhabib. Obwohl Benhabib die Bedeutung von Heideggers »In-der-Welt-Sein« für Arendts pluralistische Theorie des Politischen ausführlich würdigt, ignoriert sie den Zusammenhang zwischen dieser Grundlegung von Arendts Politikbegriff und der existentialphilosophischen Kritik am neuzeitlichen Subjektivitäts- und Rationalitätsbegriff, um dann über den vermeintlichen »anthropologischen Universalismus « Arendts diese doch recht umstandslos in eine von Kant bis zu neueren Diskurstheorien reichende Traditionslinie zu stellen. Noch bemerkenswerter scheint mir, dass das Buch an keiner Stelle auf die offensichtlichen Parallelen zwischen Arendts Kritik der Moderne und derjenigen der ersten Generation der Frankfurter Schule eingeht (vgl. Seyla Benhabib, The Reluctant Modernism of Hannab Arendt, Thousand Oaks 1996).

5 Jürgen Habermas, »Die Verschlingung von Mythos und Aufklärung. Bemerkungen zur Dialektik der Aufklärung - nach einer erneuten Lektüre« in: Karl Heinz Bohrer (Hg.), Mythos und Moderne, Frankfurt a.M. 1983, S. 429. Eine wirklich erstaunliche Kombination: hemmungslos skeptisch!

6 Vgl. dazu etwa Fritz Stern, Kulturpessimismus als politische Gefahr, Bern 1963. Speziell zum Einfluss der religiös orientierten russischen Kritik der Moderne auf das kulturkritische Denken in Deutschland auch Harald Bluhm, »Dostojewski und Tolstoi-Rezeption auf dem ssemantischen Sonderweg<. Kultur und Zivilisation in deutschen Rezeptionsmustern Anfang des 20. Jahrhunderts« in: Politische Vierteljabresschrift, 40. Jg., 2/1999, S. 305- 327. 
Wolfgang Mommsen und Jürgen Habermas zeigen konnten. Und auch Weber sieht in der Demokratie kein Symptom der Dekadenz oder Krise, sondern einen Ausweg aus dem durch Versachlichung und Bürokratisierung drohenden Sinn- und Freiheitsverlust. Schließlich orientieren sich Weber wie Arendt an der westlichen, insbesondere der amerikanischen politischen Erfahrung. Wenn es um die jeweiligen Politik- und Demokratiekonzeptionen selbst geht, sind die Parallelen allerdings erschöpft. Arendt und Weber vertreten hier unterschiedliche, ja geradezu für die entgegengesetzten Pole der zeitgenössischen Demokratietheorie stehende Vorstellungen. Meine These lautet nun, dass Webers Plädoyer für die plebiszitäre Führerdemokratie auf der einen und Arendts Eintreten für eine republikanische, partizipatorische Demokratie auf der anderen Seite sich nur durch die verschiedenen theoretischen Grundlagen ihrer jeweiligen Kulturkritik erschließen lassen. Dabei möchte ich zeigen, wie Webers sozialwissenschaftliche Theorie der Rationalisierung mit großer Konsequenz in die herrschaftliche Perspektive einer plebiszitären Führerdemokratie mündet, während Arendts existentialphilosophische und sozialwissenschaftlich gewiss nicht unproblematische Unterscheidung von Grundtätigkeiten es ermöglicht, an die in den Revolutionen der Neuzeit gemachte Erfahrung des Handelns Gleicher unter Bedingungen der Kontingenz anzuschließen. Im Ergebnis dieses Vergleiches wird m.E. auch klarer, weshalb der Webers Rationalisierungstheorie aufgreifende westliche Marxismus und insbesondere die Aufklärungs- und Vernunftkritik der Frankfurter Schule im Gegensatz zu Hannah Arendt nicht in der Lage waren, eine positive Konzeption demokratischer Politik hervorzubringen.

Ich werde dazu zunächst auf Webers Doppelthese vom Sinn- und Freiheitsverlust eingehen, um dann darzustellen, wie sehr sein politisches Denken, insbesondere sein Dezisionismus und sein herrschaftszentrierter Politikbegriff daraus hervorgehen. Danach werde ich Arendts Kritik der Moderne unter den selben beiden Leitbegriffen des Sinn- und Freiheitsverlustes zusammenfassen, um von daher zu erläutern, weshalb sie zu einem gänzlich anderen Politik- und Demokratiebegriff kommen konnte als Max Weber. Abschließend werde ich dann Webers Dezisionismus und Arendts Konzeption des Urteilens als Alternativen der Kontingenzbewältigung in modernen Gesellschaften darstellen.

7 Vgl. etwa Wilhelm Hennis, Max Webers Fragestellung, Tübingen 1987; Lawrence A. Scaff, Fleeing the Iron Cage. Culture, Politics and Modernity in the Thought of Max Weber, Berkeley 1989; Jürgen Habermas, Theorie des kommunikativen Handelns, Bd. I und II, Frankfurt a.M. 1981; Wolfgang Mommsen, Weber und die deutsche Politik, 1890-1920, Tübingen 1959. 


\section{Sinn- und Freibeitsverlust bei Max Weber}

\subsection{Sinnverlust als Transzendenzverlust}

Nachdem Weber lange Zeit vor allem als Begründer einer empirisch orientierten »wertfreien « und strukturalistisch-funktionalen Sozialwissenschaft wahrgenommen worden war, rückte während der achtziger Jahre Webers kulturtheoretisches Interesse, seine Frage nach der »Entwicklung des Menschentums« unter Bedingungen gesellschaftlicher Rationalisierung stärker in den Vordergrund. Hier sind insbesondere die bereits erwähnten Bücher von Wilhelm Hennis und Lawrence Scaff zu nennen. In der von Georg Lukács geprägten marxistischen Rezeption wurde Weber allerdings immer schon als ein Theoretiker gelesen, der den Herrschaftscharakter gesellschaftlicher Rationalisierung offen legt und, analog zur Marxschen Verdinglichungstheorie, ihre Verselbständigung gegenüber den handelnden Menschen beklagt $^{8}$. Jürgen Habermas hat in seinem Hauptwerk 1981 die Kritik Webers am Rationalisierungsprozess westlicher Gesellschaften unter der Formel vom Sinn- und Freiheitsverlust zusammengefasst ${ }^{9}$.

Am offensichtlichsten zeigt sich dieser kulturkritische Weber in den religionssoziologischen Aufsätzen. Aber auch in den politischen Schriften und den Aufsätzen zur Wissenschaftslehre nehmen die Fragen nach Menschentyp und Lebensweise einen prominenten Stellenwert ein.

Fragen wir zunächst einmal, was genau Max Weber mit der zeitkritischen Diagnose des Sinnverlustes meinte. Legen wir seine allgemeine Definition von "Sinn« als subjektive Handlungsabsicht zugrunde $^{10}$, ist nicht recht einzusehen, weshalb moderne Gesellschaften Sinnverlust hervorbringen sollten. Schließlich zeichnen sie sich nach gängiger Auffassung ja gerade durch die Erweiterung subjektiver Handlungsmöglichkeiten aus.

Eine erste Antwort ergibt sich aus dem Kontext, in dem die These des Sinnverlustes mehrfach auftaucht, nämlich aus Webers religionssoziologischen Schriften. Sinnverlust entsteht hier aus der Spannung zwischen religiöser Weltdeutung und empirischer Welt, genauer: er ist das paradoxe Resultat des religiösen Bemühens, Leben und Welt einen einheitlichen Sinn zuzuschreiben und die Lebensführung danach auszurichten. Religionen erheben nach Weber den Anspruch, »dass der Weltverlauf, wenigstens soweit er die Interessen der Menschen berührt, ein irgendwie sinnvoller

8 Zur marxistischen, durch Lukács geprägten Weberrezeption vgl. etwa Jürgen Habermas, aaO. (FN 7), Bd. I und II sowie Michael Greven, »Krise der objektiven Vernunft. Entfremdung und ethischer Dezisionismus bei Georg Lukács und Max Weber « in: U. Bermbach / G. Trautmann (Hg.): Georg Lukács, Opladen 1987, S. 97-123.

9 In dieser zusammengezogenen Formulierung findet sich die These meines Wissens nicht bei Weber. Habermas bringt damit jedoch die Hauptintentionen der Weberschen Rationalisierungskritik zutreffend auf den Punkt. Vgl. Jürgen Habermas > aaO. (FN 7), Bd. I, S. 333.

10 Max Weber, Wirtschaft und Gesellschaft (WuG), Tübingen 1947 (1925), S.1. 
Vorgang sei «11. Diesem Anspruch steht jedoch die empirische Welt mit ihrer ethisch unmotivierten Ungleichverteilung von Glück und Leid entgegen. Religionen versuchen, auf diese als Theodizee-Problem bekannte Schwierigkeit rationale Antworten zu geben - das ist der Grund, weshalb sie für Weber eine entscheidende Rolle im universalhistorischen Prozess der Rationalisierung spielen. Allerdings gelingt es ihnen nicht, die religiös begründete Ethik mit der Realität der Welt zu versöhnen, und zwar insbesondere nicht mit den Zwängen der wirtschaftlichen Welt. Nach Weber führt das Bemühen, den Konflikt zwischen dem rationalen Anspruch der Religion und der unvollkommenen, ungerechten und vergänglichen Wirklichkeit zu lösen, vielmehr zu einer immer weiteren Entwertung der Welt einerseits sowie einem immer unweltlicheren, dem Leben fremden Inhalt des Religiösen andererseits ${ }^{12}$. Einen letzten, großangelegten Versuch, hier einen Ausweg zu weisen, sieht Weber in der protestantischen Ethik, genauer in der puritanischen Berufsethik. Sie löst die Spannung zwischen den religiösen Brüderlichkeitsgeboten und den Erfordernissen rationalen Wirtschaftens auf, indem sie letztere zur Wirkstätte des göttlichen Willens umdeutet. Diese Umdeutung wird möglich, indem die puritanische Berufsethik

»...auf den Universalismus der Liebe verzichtete, alles Wirken in der Welt als Dienst in Gottes, in seinem letzten Sinn ganz unverständlichen, aber nun einmal allein erkennbaren positiven Willen und Erprobung des Gnadenstandes rational versachlichte und damit auch die Versachlichung des mit der ganzen Welt als kreatürlich und verderbt entwerteten ökonomischen Kosmos als gottgewollt und Material der Pflichterfüllung hinnahm. Das war im letzten Grunde der prinzipielle Verzicht auf Erlösung als ein durch Menschen und für Menschen erreichbares Ziel zugunsten der grundlosen, aber stets nur partikulären Gnade «13.

Dieser "Standpunkt der Unbrüderlichkeit« stellt für Weber zwar keine eigentliche Erlösungsreligion mehr dar ${ }^{14}$. Dennoch vermochte es die puritanische Berufsethik, der Askese der modernen, auf spezialisierte Facharbeit beschränkten Berufsarbeit einen höheren Sinn zu geben. Zwar sei die »faustische Allseitigkeit des Menschentums $\aleph^{15}$ oder die Freude des mittelalterlichen Handwerkers an dem, was er schuf, nun endgültig dahin ${ }^{16}$. Diesen Verlust des diesseitigen weltlichen Reizes der Arbeit könne die puritanische Berufsethik jedoch durch den Gewinn einer unmittelbaren jenseitigen Orientierung der Arbeitsaskese kompensieren:

»Die berufliche Arbeit als solche ist gottgewollt. Die Unpersönlichkeit der heutigen Arbeit: ihre, vom Standpunkte des Einzelnen aus betrachtet, freudlose Sinnlosigkeit, ist hier noch religiös verklärt. Der Kapitalismus in der Zeit seiner Entste-

11 Max Weber, "Zwischenbetrachtung: Theorie der Stufen und Richtungen religiöser Weltablehnung in: Gesammelte Aufsätze zur Religionssoziologie, Tübingen 1920, S. 567.

12 Vgl. ebd., S. 567ff.

13 Ebd., S. 545f.

14 Ebd., S. 546.

15 Max Weber, Die protestantische Ethik I. Eine Aufsatzsammlung. Hrsg. von Johannes Winckelmann, Tübingen $1981^{6}$ (1920), S. 187.

16 Ebd., S. 274, Anm. 299. 
hung brauchte Arbeiter, die um des Gewissens willen der ökonomischen Ausnutzung zur Verfügung standen. Heute sitzt er im Sattel und vermag ihre Arbeitswilligkeit ohne jenseitige Prämissen zu erzwingen « ${ }^{17}$.

Die »freudlose Sinnlosigkeit «, von der Weber hier spricht, ist keineswegs auf die unter kapitalistischen Ausbeutungsverhältnissen verausgabte Arbeit beschränkt. In einer bekannteren, immer wieder zitierten Stelle desselben Textes führt Weber allgemeiner aus, dass der religiöse Geist längst aus dem »stahlharten Gehäuse« der von ihm mit hervorgebrachten Wirtschaftsordnung entwichen $\operatorname{se}^{18}{ }^{18}$. Der einmal auf eigener Grundlage etablierte, oder wie Weber schreibt, der »siegreiche Kapitalismus" bedarf der religiösen Stütze eines jenseitigen Zweckes jedoch nicht mehr, er kann schließlich sogar auf die »rosige Stimmung der Aufklärung « der »lachenden Erbin« des religiösen Geistes verzichten ${ }^{19}$. In den kapitalistischen Gesellschaften des Westens gewinnt damit die Durchsetzung formaler, d.h. von der Bindung an bestimmte Zwecke gelösten Rationalität eine eigene, von ihren religiösen Ursprüngen unabhängige Dynamik. In Formulierungen, die nicht zufällig an Marx erinnern, spricht Weber von der "zunehmenden und unentrinnbaren Macht« der »äußeren Güter dieser Welt« über den Menschen ${ }^{20}$.

Der moderne Kapitalismus kann demnach zwar wirtschaftlich rationales Verhalten erzwingen, aber er ist immer weniger in der Lage, ihm einen höheren Sinn zu geben.

»Auf dem Gebiet seiner höchsten Entfesselung, in den Vereinigten Staaten, neigt das seines religiös-ethischen Sinnes entkleidete Erwerbsstreben dazu, sich mit rein agonalen Leidenschaften zu assoziieren, die ihm nicht selten den Charakter des Sports aufprägen $\ll^{21}$.

Im Anschluss an dieses Zitat findet sich Webers berühmte Warnung vor den »Fachmenschen ohne Geist, Genussmenschen ohne Herz« als Kulminationspunkt der Kulturentwicklung des Westens ${ }^{22}$.

Sinnverlust entsteht durch die Verselbständigung der Ökonomie gegenüber religiösen Weltbildern und den Handlungsorientierungen, die der einzelne Mensch aus ihnen gewinnen konnte. Er steht am Ende des universalgeschichtlichen Prozesses der »Entzauberung der Welt« und ihrer Verwandlung in einen kausalen Mechanismus, der nicht mehr als "gottgeordneter, also irgendwie ethisch sinnvoll geordneter Kosmos « wahrzunehmen ist ${ }^{23}$. Zurück bleibt ein äußerlich gewordener $Z_{w a n g}$, »Versteinerung «, »Mechanisierung « und »tote Maschinerie«, wie die immer wieder benutzten Metaphern Webers für die unpersönliche Herrschaft der rationalisierten gesellschaftlichen Verhältnisse über die Individuen lauten.

17 Ebd., S. 275, Hvhbg. von Max Weber.

18 Ebd., S. 188.

19 Ebd.

20 Ebd.

21 Ebd., S. $188 f$.

22 Ebd., S. 189.

23 Vgl. Max Weber, Zwischenbetrachtung, aaO. (FN 11), S. 564. 
Hervorzuheben ist, dass Weber hier nicht etwa zweckrationales Handeln kritisiert, sondern im Gegenteil die Emanzipation des ökonomischen Handelns aus der Unterordnung unter letzte, religiös begründete Zwecke. Die Kategorie der Zweckrationalität ist bei Weber auch im Zusammenhang seiner Kulturkritik nicht negativ besetzt ${ }^{24}$. Sinn und Zweck sind für Weber identisch. Was ihn beunruhigt, ist die Durchsetzung formaler Rationalität in den Apparaten der Produktion und der Bürokratie. Formale Rationalisierung meint einen Prozess der Durchstrukturierung, Logifizierung und Systematisierung von Ordnungen und Handlungen ${ }^{25}$, kurz des Beherrschbar- und Berechenbarmachens ohne Bindung an bestimmte Zwecke. Man könnte auch sagen: formal rationalisierte Organisationen befreien sich von letzten Zwecken, verkörpern höchste Zweckmäßigkeit ohne Zweck.

\subsection{Sinnverlust als Verlust der Einheitlichkeit der Welt}

Sinnverlust und »Entzauberung der Welt« meinen bei Weber jedoch nicht nur den Verlust religiöser Transzendenz, sondern mit ihr zugleich auch den Verlust der Einheitlichkeit der Welt und damit der Möglichkeit, die Lebensführung ethisch-methodisch an einer religiös begründeten Zweckhierarchie auszurichten. Denn Rationalisierung bedeutet »Herauspräparierung der spezifischen Eigenart jeder in der Welt vorkommenden Sondersphäre ${ }^{26}$. Es entstehen verschiedene Wertsphären, etwa der Ökonomie, der Kunst, der Ethik. Mit Hinweis auf Baudelaires »Fleurs du mal« unterstreicht Weber, dass etwas schön sein kann, nicht nur ohne gut zu sein, sondern sogar gerade in dem, worin es nicht gut ist ${ }^{27}$. Für Weber handelt es sich hier jedoch nicht nur um die Verselbständigung von Wertsphären, die untereinander wiederum in ein Ergänzungs- oder Kompromissverhältnis zu setzen wären. Explizit betont er, es gehe hier nicht nur um Alternativen, sondern um »unüberbrückbar tödlichen Kampf « ${ }^{28}$. In diesem agonalen Verhältnis gründet für Weber die Unmöglichkeit, praktische Stellungnahmen wissenschaftlich zu vertreten und damit zugleich die Unmöglichkeit, über letzte Zwecke in irgendeiner Weise rational zu entscheiden. Nachdrücklich wendet er sich gegen die Vorstellung, das Abwägen von Gründen und ethischen Prinzipien könne derartige Entscheidungen rationalisieren ${ }^{29}$.

24 So formuliert er in seinem berühmten »Objektivitätsaufsatz«: »Jede denkende Bestimmung auf die letzten Elemente sinnvollen menschlichen Handelns ist zunächst gebunden an die Kategorien 'Zweck< und >Mittek» (Max Weber, Gesammelte Aufsätze zur Wissenschaftslebre (GAW), Tübingen $1988^{7}$ (1922), S. 149).

25 Vgl. dazu Stefan Breuer, Bürokratie und Charisma. Zur politischen Soziologie Max Webers, Darmstadt 1994, S. $39 \mathrm{ff}$.

26 Vgl. Max Weber, Zwischenbetrachtung, aaO. (FN 11), S. 571.

27 Max Weber, GAW, aaO. (FN 24), S. 604.

28 Ebd., S. 507.

29 Ebd., S. 507ff., 602ff. Ausführlicher zu der damit verbundenen Ablehnung der praktisch-philosophischen Tradition durch Weber vgl. Hella Mandt, Tyrannislebre und Widerstandsrecht, Darmstadt 1974, S. $267 \mathrm{ff}$. 
Ohne Möglichkeit, sich auf eine einheitliche Weltanschauung zu beziehen und aus ihr eine ethisch-methodische Lebensführung abzuleiten, bleibt dem auf sich zurückgeworfenen Individuum nur, dieses »Schicksal der Zeit«»männlich zu ertragen « und sich zwischen den letzten Standpunkten zum Leben zu entscheiden ${ }^{30}$. Individueller Heroismus und ethischer Dezisionismus treten bei Weber an die Stelle eines religiös verbürgten einheitlichen Sinnes.

Wie Wilhelm Hennis überzeugend darstellen konnte, gilt Webers zentrales Interesse der »Entwicklung des Menschentums « ${ }^{31}$. Ihn beunruhigt die Verdrängung des »Kulturmenschen« durch den »Ordnungs «- oder »Berufsmenschen« bzw. den zweifach, als »Fachmenschen ohne Geist« und als »Genussmenschen ohne Herz« bestimmten Typus der Zukunft ${ }^{32}$. Weber entwickelt sein Gegenmodell, das Individuum als verantwortungsbewusste Persönlichkeit, aus der oben beschriebenen Entscheidungssituation. Unter den Bedingungen einer mechanisierten und in Wertsphären zerfallenden Gesellschaft wählt die Persönlichkeit den Sinn ihres Tuns und Seins selbst ${ }^{33}$. Allerdings qualifiziert Weber diesen voluntaristischen Akt in Abgrenzung zur subjektivistischen Kultur der Moderne, indem er von ihr Konsequenz bzw. Hingabe an die einmal gewählte Sache sowie intellektuelle Rechenschaftspflicht, also Reflexivität fordert ${ }^{34}$. Dies bildet den Hintergrund für Webers Verantwortungsethik, die sich im klaren Bewusstsein der praktischen Folgen und der Rationalität der Mittel im Verhältnis zum gewählten Zweck zu erweisen hat ${ }^{35}$. In der subjektivistischen Kultur der Moderne mit ihrer Suche nach authentischer Erfahrung und dem »Jagen nach Erlebnis« kann Weber dagegen nur eine "Schwäche«, gewissermaßen die Kehrseite der gesellschaftlichen Mechanisierung erkennen ${ }^{36}$. Für den Gewinn persönlicher Freiheit muss, ähnlich wie im Puritanismus, der Preis der Askese und Selbstbeherrschung entrichtet werden. Handeln ist für Weber umso freier, je mehr es den Charakter eines naturhaften Geschehens ablegt und in der Konstanz eines persönlich gewählten Verhältnisses zu letzten Werten und Bedeutungen steht ${ }^{37}$.

\subsection{Freibeitsverlust}

Damit ist nun allerdings die Frage nach dem zweiten Element in Webers Kulturkritik, dem Freiheitsverlust, aufgeworfen. In den bereits zitierten Metaphern vom »stahlharten Gehäuse« oder den »vielen alten Göttern«, die nach Gewalt über unser

30 Max Weber, GAW, aaO. (FN 24), S. 612, S. 608.

31 Wilhelm Hennis, Max Webers Fragestellung, Tübingen 1987, S. 20.

32 Max Weber, Die protestantische Ethik I, aaO. (FN 15), S. 189.

33 Vgl. Max Weber, GAW, aaO. (FN 24), S. 180 und S. 508.

34 Ebd., S. 494 und S. 608.

35 Vgl. Max Weber, Gesammelte Politische Schriften (GPS), Tübingen $1988^{5}$ (1921), S. $551 f$.

36 Max Weber, GAW, aaO. (FN 24), S. 605.

37 Vgl. ebd., S. 132. 
Leben streben, spricht Weber Freiheitsverlust als Folge der Verselbständigung gesellschaftlicher Organisationen und ihrer Funktionsweise an. Und zwar verselbständigen sich insbesondere kapitalistische Ökonomie und staatliche Bürokratie gegenüber den Individuen und deren moralisch-praktischen Handlungsmotiven. Es ist also zunächst einmal zu unterstreichen, dass Weber nicht nur in der Bürokratisierung, sondern auch im Siegeszug der kapitalistischen Ökonomie eine Tendenz zur Zerstörung individueller Freiheit sieht. Dem »heutigen Hochkapitalismus « eine »Wahlverwandtschaft mit >Demokratie oder gar mit `Freiheit (in irgend einem Wortsinn) zuzuschreiben«, bezeichnet er als »höchst lächerlich « ${ }^{38}$. Im Gegensatz zu den Fortschrittshoffnungen seiner Zeit (und in einem nicht minderen zu heutigen neoliberalen Vorstellungen) erwartet er als Ergebnis ökonomischer Vergesellschaftung keinen Freiheitsgewinn, sondern eine Einschränkung der Persönlichkeits- und Freiheitssphäre des Individuums. Die modernen Vorstellungen von Freiheit, Individualismus und demokratischen Institutionen sind für ihn das Ergebnis einzigartiger, sich nicht wiederholender historischer Konstellationen in der westlichen Welt ${ }^{39}$, keineswegs das Ergebnis gesellschaftlicher Rationalisierung. Sie müssen deshalb auch »wider den Strom « der materiellen Entwicklungstendenzen erobert bzw. gesichert werden ${ }^{40}$.

Freiheitsverlust bildet für Weber schon deshalb die unausweichliche Kehrseite gesellschaftlicher Rationalisierung, weil sowohl rationales Wirtschaften wie auch rationale Verwaltung erst durch Herrschaftsverhältnisse möglich werden. Die sozialstrukturelle Voraussetzung von Webers formaler Rationalität bildet »die Trennung des Arbeiters von den sachlichen Produktionsmitteln in der Wirtschaft, von den Kriegsmitteln im Heer, den sachlichen Verwaltungsmitteln in der öffentlichen Verwaltung ${ }^{41}$. Gesellschaftliche Rationalisierung kann demnach nur in dem Maße voranschreiten, wie die Arbeitenden, oder allgemeiner die rational Tätigen, die Möglichkeit verlieren, ihr Handeln nach eigenen Zielvorstellungen auszurichten und gezwungen werden können, als Teil einer kalkulierbaren Maschinerie zu funktionieren.

Insbesondere die Bürokratie charakterisiert Weber immer wieder als Maschine oder als Mechanismus. Die formal rationale Bürokratie basiert auf straffer arbeitsteiliger Organisation, hierarchisch abgestuften Gehorsamsverhältnissen, fachlicher Schulung und vor allem auf einer sachlichen Erledigung ihrer Aufgaben nach berechenbaren Regeln ${ }^{42}$. Diese regelgebundene »kühle Sachlichkeit« funktioniert ohne »Ansehen der Person« und kann mit materialen Gerechtigkeitsvorstellungen kollidieren $^{43}$, ganz ähnlich wie die kapitalistische Geld- und Gewinnrechnung sich von

38 Max Weber, GPS, aaO. (FN 35), S. 64.

39 Neben religiös bestimmten Wertvorstellungen zählt Weber hierzu vor allem die überseeische Expansion der frühen Neuzeit, den anarchischen Charakter des Frühkapitalismus und die Vorstellung von einer wissenschaftlich gebildeten universellen Persönlichkeit (vgl. Max Weber, GPS, aaO. (FN 35), 64f.).

40 Ebd., S. 65.

41 Ebd., S. 322.

42 Vgl. ebd., S. 332; Max Weber, $W u G$, aaO. (FN 10), S. 661.

43 Max Weber, WuG, aaO. (FN 10), S. 664. 
gesellschaftlichen Bedürfnissen und Produktionszielen lösen kann. Auf die Funktionszwänge einer solchen Bürokratie bezieht Weber sein berühmtes »Gehäuse der Hörigkeit«, in welches

»vielleicht dereinst die Menschen sich, wie die Fellachen im altägyptischen Staat, ohnmächtig zu fügen gezwungen sein werden, wenn ihnen eine rein technisch gute und das heißt: eine rationale Beamtenverwaltung der letzte und einzige Wert ist, der über die Leitung ihrer Angelegenheiten entscheiden soll «4

Es handelt sich bei diesem Zitat allerdings weder um eine Zustandsbeschreibung noch um eine Prophetie. Weber sagt »wenn ...«, es gibt also Alternativen und Möglichkeiten, das »Gehäuse der Hörigkeit« zu vermeiden.

\subsection{Webers Lösung: Dezision und Herrschaft}

Es ist diese Rückführung formaler Rationalität auf ein gesellschaftliches Herrschaftsverhältnis, das Georg Lukács die Möglichkeit bot, Webers Rationalisierungstheorie marxistisch umzuformulieren. Wenn Rationalität ein Klassenverhältnis voraussetzt, dann muss sie sich auch - so die logische Schlussfolgerung - mit diesem Klassenverhältnis aufheben lassen. Für Lukács ist Webers Durchsetzung formaler Rationalität das Ergebnis der voranschreitenden Subsumtion der Gesellschaft unter die Wertabstraktion kapitalistischer Warenproduktion. Sie enthält damit den Widerspruch zwischen Gebrauchswert und Tauschwert, zwischen konkreter und abstrakter Arbeit oder politisch revolutionär gewendet, den Widerspruch der Arbeiter gegen den (ihnen aufgezwungenen) Warencharakter ihrer Arbeitskraft.

Am immanent widersprüchlichen Charakter gesellschaftlicher Rationalisierung hält übrigens auch Jürgen Habermas fest. Er erweitert dazu allerdings das Verständnis gesellschaftlicher Rationalisierung um den Begriff der kommunikativen Verständigung und gibt die Vorstellung von einer revolutionären Klasse auf, die als Verkörperung der materialen Vernunft bedürfnisorientierter Produktion gelten kann. In den Kategorien von Habermas lässt sich der funktionalen Vernunft in den ausdifferenzierten Subsystemen der Ökonomie und des Staates das durch die Rationalisierung von Lebenswelten freiwerdende Potential verständigungsorientierten Handelns entgegensetzen ${ }^{45}$. An die Stelle des schicksalhaften Freiheits- und Sinnverlustes bei Max Weber tritt dann die Gefahr des kolonialisierenden Übergriffs der Steuerungsmedien Geld und Macht auf die Lebenswelt, eine Gefahr gesellschaftlicher Rationalisierung, zur der Habermas nun jedoch in der posttraditionalen Kommunikation moderner Gesellschaften eine ebenfalls rationale Gegenkraft identifiziert.

Wie wir wissen, konnte Weber in der Rationalisierung moderner Gesellschaften keine immanente Dialektik entdecken, weder in revolutionär-sozialistischer noch in evolutionär-demokratischer Form. Ausdrücklich verneint er die Möglichkeit, dass »irgendeine materielle oder gar die heutige hochkapitalistische Entwicklung « die

44 Max Weber, GPS, aaO. (FN 35), S. 332.

45 Vgl. etwa Jürgen Habermas, aaO. (FN 7), II, S. $485 \mathrm{ff}$. 
Bedingungen individueller Freiheit schaffen oder auch nur erhalten könne «46. Bekannt ist auch Webers Urteil über die sozialistischen Bewegungen seiner Zeit. Obwohl er im Sozialismus eine kulturkritische Bewegung sah, der es in erster Linie darum ging, die bürgerliche Welt mit neuen Werten zu konfrontieren, prophezeite er für den Fall ihres Erfolges eine Verstärkung der freiheitszerstörenden Tendenzen moderner Gesellschaften ${ }^{47}$. Die Ausschaltung des Privatkapitalismus führe lediglich zu einer Alleinherrschaft der staatlichen Bürokratie und mache somit das »stählerne Gehäuse der Hörigkeit« nur noch undurchdringlicher ${ }^{48}$.

Weber sucht die Gegenkräfte zum diagnostizierten Sinn- und Freiheitsverlust außerhalb seines Rationalitätsbegriffes in den voluntaristischen wertsetzenden Entscheidungen des Individuums. Politisch bedeutsam wird diese bereits oben charakterisierte Perspektive dadurch, dass Weber insbesondere zwei Gruppen für persönlich qualifiziert und aufgrund ihrer gesellschaftlichen Stellung auch für fähig hält, als soziale Träger freiheitssichernder Dezision zu wirken: kapitalistische Unternehmer und politische Führer ${ }^{49}$. Beide verkörpern für Weber das, was der rationalen Verwaltung, sei es im Wirtschaftsbetrieb, sei es im Staat, fehle, nämlich den zur eigenverantwortlichen Entscheidung fähigen »leitenden Geist « ${ }^{50}$. Für uns ist dabei in erster Linie der politische Führer von Interesse. Das galt übrigens aber auch für Weber selbst, weil er anders als heutige Systemtheoretiker in der Politik noch das Zentrum der Gesellschaft sah.

Im Folgenden möchte ich zeigen, dass dieses dezisionistische Heilmittel gegen die Entfremdungserscheinungen moderner Gesellschaften a) formal und b) pessimistisch-elitär ist sowie c), worauf es mir hier besonders ankommt, den versachlichten Verhältnissen moderner Gesellschaften eine Vorstellung von direkter, durch Befehls-Gehorsamsverhältnisse bestimmter Herrschaft entgegensetzt und zwar auch da, wo Weber für die Demokratisierung Deutschlands nach westlichem, insbesondere amerikanischem Vorbild eintritt.

a) Die Fähigkeit zu eigenverantwortlichem Handeln ist für Weber nicht nur persönliche Begabung, sondern mehr noch das Ergebnis von Sozialisationsprozessen. Unternehmer und Politiker stehen im Kampf um ihre eigene Sache und unterscheiden sich damit vom modernen Durchschnittsmenschen, der längst zu einem »Rädchen « in Wirtschaft und Verwaltung wurde »und innerlich zunehmend darauf abgestimmt (ist), sich als ein solches zu fühlen und sich nur zu fragen, ob er nicht von diesem kleinen Rädchen zu einem größeren werden kann ${ }^{51}$.

Dass gerade Unternehmer und Politiker Webers Ideal des Kulturmenschen retten sollten, mag erstaunen. Aber Weber kommt es hier nicht auf die Inhalte unterneh-

46 Max Weber, GPS, aaO. (FN 35), S. 65.

$47 \mathrm{Zu}$ Webers Auffassung vom Sozialismus als kulturkritischer Bewegung s. Lawrence A. Scaff, Fleeing the Iron Cage, aaO. (FN 7), S. $175 \mathrm{ff}$.

48 Vgl. Max Weber, GPS, aaO. (FN 35), S. $331 \mathrm{f}$.

49 Ebd., S. 334.

50 Ebd.

51 Max Weber, Gesammelte Aufsätze zur Soziologie und Sozialpolitik(GASS), Tübingen 1924, S. 413. 
merischer oder politischer Tätigkeit an, sondern rein formal auf den Gesichtspunkt der eigenverantwortlichen Entscheidung, zu der weder Pflichtbewusstsein noch Fachwissen, sondern nur die Erfahrung des Machtkampfes in eigener Sache befähige. »Kampf um eigene Macht und die aus dieser Macht folgende Eigenverantwortung für seine Sache ist das Lebenselement des Politikers wie des Unternehmers «52. Freiheit ist hier gedacht als Autonomie der Entscheidung, Autonomiefähigkeit als Ergebnis einer Schule des »Kampfes«.

b) Webers Kulturkritik oszilliert zwischen der pessimistischen Beschreibung unaufhaltsamer, schicksalhafter Tendenzen der gesellschaftlichen Versachlichung und dem Pathos, mit dem er dazu aufruft, gegen die große Maschinerie moderner Gesellschaften »noch einen Rest des Menschentums freizuhalten von dieser Parzellierung der Seele, von dieser Alleinherrschaft des bürokratischen Ideals « ${ }^{53}$. Darauf bezogen spricht Greven von »tragisch-heroischen Zügen« in der Haltung Webers, von einer »historischen Rückzugsposition«, oder auch vom »Konzept einer historisch defensiven Elite « ${ }^{54}$.

Obwohl Weber in Unternehmern und politischen Führern soziale Träger der Gegenkräfte zu den freiheitszerstörenden Tendenzen der Moderne bestimmt, seine Kulturkritik also nicht auf Appelle beschränkt bleibt, können diese Gegenkräfte in nennenswertem Maße nur dort entstehen, wo die Erfahrung des eigenständigen Kampfes um Macht möglich ist. Von vornherein sind sie deshalb auf Herrschaftseliten beschränkt. Da die gesellschaftliche Rationalisierung und ihr prägender Einfluss auf den Charakter der Massen unaufhaltsam voranschreitet, besteht keine realistische Aussicht darauf, die Bedingungen gesellschaftlicher Unfreiheit zu beseitigen. Es kann lediglich gehofft werden, durch die Haltung entscheidungsfähiger Eliten einen begrenzten Raum des freien, verantwortungsbewussten Handelns zu retten. Insofern bleibt Weber ganz dem elitär-pessimistischen Gestus konservativer Kulturkritik verhaftet, obwohl er ihr eine politische, demokratiebefürwortende Wendung gibt.

c) Die zitierte Formulierung Webers vom »leitenden Geist« deutet bereits darauf hin, dass er der Verselbständigung der »versteinerten Mechanik « oder der »gesellschaftlichen Maschinerie« durch eine Wiederbelebung direkter, das heißt nicht durch verselbständigte Handlungsfolgen vermittelter Herrschaftsverhältnisse ${ }^{55}$ begegnen will. Zwar gilt ihm die weitere Versachlichung gesellschaftlicher Verhältnisse als unentrinnbar. Jedoch kann die Maschinerie insgesamt, sei es der einzelne Wirtschaftsbe-

52 Max Weber, GPS, aaO. (FN 35), S. 335.

53 Max Weber, GASS, aaO. (FN 51), S. 414.

54 Michael Greven, »Krise der objektiven Vernunft. Entfremdung und ethischer Dezisionismus bei Georg Lukács und Max Weber« in: U. Bermbach / G. Trautmann (Hg.), Georg Lukács, Opladen 1987, S. 118.

55 Die Unterscheidung zwischen direkter und indirekter Herrschaft stammt aus der Debatte um den Charakter des sog. "realen Sozialismus « der siebziger Jahre. Insbesondere Renate Damus beschrieb in ihren Arbeiten mit dem Begriff der direkten Herrschaft den Versuch der kommunistischen Staats- und Parteiführungen, formale Rationalität unter Umgehung von Warenbeziehungen durch Befehls-Gehorsamsverhältnisse durchzusetzen (vgl. Renate Damus, Der reale Sozialismus als Herrschaftssystem am Beispiel $\operatorname{der} D D R$, Frankfurt a.M. 1978). 
trieb oder der bürokratisierte Staat, durch starke Führerpersönlichkeiten zur Verwirklichung bestimmter Zwecke genutzt werden. Dies ist allerdings nur in dem Maße möglich, wie es gelingt, die »Maschinerie in ein zweckrationales hierarchisches Befehls-Gehorsamsverhältnis einzufügen. Bekanntermaßen bestimmt Weber Herrschaft als »Chance, für einen Befehl bestimmten Inhalts bei angebbaren Personen Gehorsam zu finden ${ }^{\star 56}$. So definiert, eröffnen Herrschaftsverhältnisse demjenigen, der an der Spitze steht, die Perspektive einer freien Entscheidung über die Zwecke sowie ihrer Verwirklichung entlang von Befehls-Gehorsamsketten. Warum Webers Denken um das Problem der Herrschaft kreist, warum er, wie Hennis formuliert, geradezu »behext« war vom Problem der Führung und Herrschaft ${ }^{57}$, findet hier eine Erklärung in seiner Kulturkritik. Wie wir gesehen haben, führt Weber Sinnund Freiheitsverlust in modernen Gesellschaften auf die zunehmenden Schwierigkeiten zurück, das eigene Leben konsequent auf die Verwirklichung selbstbestimmter Zwecke auszurichten. Die Möglichkeit von Sinngebung und Freiheit ist also an eine zweckrationale Handlungsstruktur gebunden. Webers Lösung unter den Bedingungen einer rationalisierten Gesellschaft liegt nun darin, eine solche zweckrationale Handlungsstruktur auf höherer, politischer Ebene wiederherzustellen.

Gewiss erinnert Webers Begriff der Herrschaft mit seiner Betonung von BefehlsGehorsamsverhältnissen an die vormoderne Beziehung zwischen Herr und Knecht. Er kann auch durchaus plausibel als Ausdruck der autoritären Traditionen des preuBischen Obrigkeitsstaates interpretiert, oder spezifischer noch auf den Einfluss der deutschen Staatsrechtslehre zurückgeführt werden ${ }^{58}$. Dennoch ist Max Weber selbstverständlich kein Apologet vorbürgerlich-aristokratischer Gesellschaftsverhältnisse. Entscheidend scheint mir vielmehr, dass er der versachlichenden Rationalisierung moderner Gesellschaften ein Modell zweckrationalen Handelns gegenüberstellt, nach dem der Handelnde autonom, möglichst frei von Zwängen wie von Affekten einen $Z$ weck wählt und dann, nach Maßgabe der Situation, die zur Erreichung seiner Zwecke geeigneten Mittel bestimmt ${ }^{59}$. Der autonome Akt der Dezision und das distanzierte zweckrationale Kalkül bilden übrigens, wie Weber selbst betont, einen entscheidenden Unterschied zu romantischen Vorstellungen persönli-

56 Max Weber, WuG, aaO. (FN 10), S. 28.

57 Wilhelm Hennis, Max Webers Fragestellung, aaO. (FN 7), S. 219.

58 Im ersten Sinn etwa Dolf Sternberger, »Max Weber und die Demokratie« in: Herrschaft und Vereinbarung, Frankfurt a.M.1986 oder Rigby, der Herrschaft als »earthy German word « bezeichnet und den Herrschaftsbegriff wegen seiner Nähe zu agrarisch-feudalen Verhältnissen für ungeeignet hält, moderne politische Systeme zu analysieren, die auf Verhandlungen, Kompromissen etc. basieren (T.H. Rigby, »Introduction: Political Legitimacy, Weber and Mono-organisational Systems « in: T.H. Rigby / Ference Fehér (eds.), Political Legitimation in Communist States, London/Basingstoke 1982, S. 7). Spezifischer zum Einfluss der deutschen Staatsrechtslehre auf Weber etwa Ernst Vollrath, »Max Weber: Sozialwissenschaft zwischen Staatsrechtslehre und Kulturkritik in: Politische Vierteljabresschrift, 31. Jg., 1/1990, S. 102-108.

59 Vgl. Max Weber, GAW, aaO. (FN 24), S. 132f. Dieses Handlungsmodell lässt sich mit Habermas als teleologisch und monologisch charakterisieren (vgl. Jürgen Habermas, Theorie des kommunikativen Handelns, aaO. (FN 7) Bd. I, S. 378). 
cher Freiheit ${ }^{60}$, aber auch zur Position Nietzsches, der Zweckrationalität als Quelle des Nihilismus kritisiert ${ }^{61}$. Unschwer ist in diesem teleologischen Handlungsmodell die neuzeitliche Vorstellung von Willensfreiheit und Souveränität zu erkennen. Ebenso eindeutig ist aber auch seine Nähe zur Gewalt, wo immer es auf den Bereich des Politischen bezogen wird. Denn die Fähigkeit, eigene Ziele durchzusetzen, ist umso größer, je effektiver ich die anderen, mit denen ich den politischen Raum teile, von der Zielbestimmung ausschließen und zur Verwirklichung meiner Zwecke instrumentalisieren kann. Dem teleologischen Handlungsmodell entspricht ein Verhältnis zwischen Befehlenden und Gehorchenden. Wie Arendt in ihrer expliziten Auseinandersetzung mit Webers Herrschaftsbegriff in »Macht und Gewalt« zugesteht, ist der wirkungsvollste Befehl zwar derjenige, der mit Gewalt drohen kann. Allerdings, so ihr Einwand, zerstört die auf Gewalt gegründete Fähigkeit, die eigenen Ziele auch gegen Widerstreben durchzusetzen, den politischen Raum und damit die in ihm entstehende, auf Zustimmung gegründete $\mathrm{Macht}^{62}$.

Gerade Webers späte politische Schriften, in denen er das wilhelminische Deutschland mit den parlamentarischen Demokratien des Westens vergleicht, lassen erkennen, wie stark sein Plädoyer für die Parlamentarisierung Deutschlands durch ein Politikverständnis geprägt ist, das Regierende und Regierte in ein arbeitsteiliges Verhältnis von Entscheidung und Ausführung stellt. Zwar würdigt er nach seiner Amerikareise von 1904 die freiwilligen, dem Vorbild religiöser Sekten folgenden Assoziationen auf kommunaler Ebene als Gegengewicht zur Atomisierung der Individuen in modernen Massengesellschaften ${ }^{63}$. Es sind letztlich aber gerade nicht diese bürgerschaftlichen Seiten der amerikanischen Demokratie, die Weber für zukunftsträchtig hält und in den Auseinandersetzungen über die politische Entwicklung Deutschlands als nachahmenswert propagiert ${ }^{64}$. Nach Weber läuft die egalitäre Logik derartiger Zusammenschlüsse der Logik der Zweckrationalität zuwider und untergräbt damit konsistente und effiziente Entscheidungen, auf die es ihm politisch gera-

60 Vgl. Max Weber, GAW, aaO. (FN 24), S. 132.

61 Dazu ausführlicher Dana R. Villa, Arendt and Heidegger. The Fate of the Political, Princeton 1996.

62 »Aus den Gewehrläufen kommt immer der wirksamste Befehl, der auf unverzüglichen, fraglosen Gehorsam rechnen kann. Was niemals aus Gewehrläufen kommt, ist Macht« (Hannah Arendt, Macht und Gewalt (MG), München 19907, (1970), S. 54).

63 Vgl. dazu Wolfgang J. Mommsen, Max Weber. Gesellschaft, Politik, Geschichte, Frankfurt a. M. 1974 und Sung Ho Kim, »In Affirming Them, He Affirms Himself. Max Weber's Politics of Civil Society « in: Political Theory, Vol. 28, 2/2000, S. 197-229. Während Mommsen dieses Element in Webers Amerikabild relativiert, interpretiert Kim Weber als Protagonisten einer aktiven Zivilgesellschaft.

64 Zum Begriff eines bürgerschaftszentrierten im Gegensatz zu einem herrschaftszentrierten Politikbegriff vgl. Jürgen Gebhardt, "Auf der Suche nach dem Politischen « in: Michael Greven / Rainer Schmalz-Bruns (Hg.), Bürgersinn und Kritik, Festschrift für Udo Bermbach zum 60. Geburtstag, Baden-Baden 1998, S. 15-27. 
de ankommt ${ }^{65}$. Statt der egalitären Formen einer bürgerschaftlichen Politik rückt Webers Blick auf das demokratische Amerika vor allem die bürokratisch organisierte Massenpartei und ihren plebiszitär-charismatischen Führer ins Zentrum des Bildes.

Weber begründet sein Eintreten für die parlamentarische Regierungsform während des Ersten Weltkrieges zunächst damit, dass sie Berufspolitiker hervorbringe, die durch den Machtkampf untereinander als starke Führer qualifiziert, d. h. zu Dezision und Verantwortung fähig seien ${ }^{66}$. In diesem Typ des Politikers sah Weber bekanntlich eine Voraussetzung der nationalen Selbstbehauptung Deutschlands ${ }^{67}$. Die Bedeutung des Parlaments für die Sozialisation und die Auslese dieser starken Führerpersönlichkeiten betont Weber während des Krieges stärker als in den Schriften, die unmittelbar danach entstanden. Sowohl in der späteren Fassung der Herrschaftssoziologie von 1919/20 als auch in »Politik als Beruf « verlagert sich der Akzent auf die plebiszitär-charismatischen Qualitäten des Führers und seine Stellung zum Parteiapparat. Es scheint, als sei Weber der parlamentarische Alltag nun zu sehr durch Routine und Betrieb bestimmt. Er traut ihm jedenfalls nicht mehr ohne weiteres zu, Politiker mit »Berufung «, d.h. mit der Fähigkeit zur Wertsetzung hervorzubringen ${ }^{68}$. Das erwartet er dagegen vom außeralltäglichen Charisma des plebiszitären Führers. Die plebiszitäre Führerdemokratie, in der die Massen einen rhetorisch begabten oder gar demagogisch agierenden Politiker ins Amt wählen, ist deshalb eher geeignet, mit dem Verhältnis von Führer und Gefolgschaft zugleich Dezision und Zweckrationalität, und damit, aus Webers Perspektive, Freiheit und Sinn in den politischen Bereich zurückzubringen ${ }^{69}$. Er macht überhaupt keinen Hehl daraus, dass diese »Führerdemokratie mit Maschine blinden Gehorsam erfordere und die »Entseelung der Gefolgschaft, ihre geistige Proletarisierung « bedin$\mathrm{ge}^{70}$. Aber das nimmt er in Kauf, weil ihm unter Bedingungen gesellschaftlicher Rationalisierung das wertsetzende Charisma des Führers der einzige Weg scheint, dem »ehernen Gehäuse der Hörigkeit« zu entkommen. Weber plädiert zu Beginn des letzten Jahrhunderts für die Demokratisierung Deutschlands, weil er die Demokra-

65 Dies zeigt Kloppenberg in einem aufschlussreichen Vergleich Webers mit Dewey (James T. Kloppenberg »Demokratie und Entzauberung der Welt: Von Weber und Dewey zu Habermas und Rorty « in: Hans Joas (Hg.), Philosophie und Demokratie, Frankfurt a.M. 2000, S. 44-80).

66 Vgl. Max Weber, GPS, aaO. (FN 35), S. 340ff., S. 364.

67 Zur Perspektive nationaler Selbstbehauptung in den politischen Schriften Webers etwa Mommsen 1959. Mommsen zitiert zustimmend Lukács, nach dem Demokratisierung für Weber eine »technische Maßnahme zugunsten eines besser funktionierenden Imperialismus" gewesen sei (Wolfgang Mommsen, Weber, aaO. (FN 7), S. 422).

$68 \mathrm{Zu}$ dieser Verschiebung ausführlich Peter Breiner, Max Weber and Democratic Politics, Ithaca/London 1996 und Wolfgang Mommsen, »Politik im Vorfeld der Hörigkeit der Zukunft. Politische Aspekte der Herrschaftssoziologie Max Webers in: Edith Hanke / Wolfgang Mommsen (Hg.), Max Webers Herrschaftssoziologie, Tübingen 2001, S. 302-319.

69 Mommsen verweist bereits 1959 auf diesen Zusammenhang, wenn er schreibt, dass Weber »den Weg der plebiszitär-charismatischen Herrschaft des großen Demagogen einschlug «, um der »Gefahr der bürokratischen Erstarrung der modernen Massengesellschaft « zu entgehen (Wolfgang Mommsen, Weber, aaO. (FN 7), S. 436). Vgl. Max Weber, GPS, aaO. (FN 35), S. 544. 
tie für geeignet hält, durch Führer-Gefolgschaftsverhältnisse die versteinerte Maschinerie der rationalisierten gesellschaftlichen und staatlichen Apparate unter die Kontrolle persönlicher Dezision zu bringen und ihr einen wertrational bestimmten Zweck vorzugeben. So gesehen ermöglichen die Demokratien des Westens mit ihren plebiszitären politischen Führerpersönlichkeiten und den ihnen untergeordneten Parteiapparaten eine zugleich herrschaftlichere und freiheitlichere Politik als das bürokratisierte Deutschland unter Wilhelm II. ${ }^{71}$.

Ausgehend von einem aristotelischen Politikverständnis wurde Max Weber vorgeworfen, seine Definitionen von Staat und Herrschaft verkehrten mit ihrer Konzentration auf Gewalt und Befehls-Gehorsamsverhältnisse das Despotische zum Politischen $^{72}$. Tatsächlich steckt in Webers Herrschaftssoziologie ein platonisches Element. In diesem Zusammenhang hat Edith Hanke jüngst darauf hingewiesen, dass Weber seinen Herrschaftsbegriff erst nach 1910 unter dem Einfluss von Georg Simmel und Georg Jellinek präzisiert habe. Dabei bemerkt sie, dass Simmel, Platon zitierend, Herrschaft auf ihren Kern zurückführe, nämlich auf »ein und diesselbe Fähigkeit, zu befehlen, die der politikos wie der basileus, der despotos wie der oikonomos besitzen müsse «73. Dennoch ist Webers Position im klassischen Gegensatz zwischen aristotelischem und platonischem Denken nicht zu fassen. Weber will Herrschaft nicht als Befehls-Gehorsamsverhältnis zwischen Wissenden und Unwissenden legitimieren. Wie bekannt, betont er ja immer wieder, die letzten wertrationalen Entscheidungen seien nicht rational begründbar. Weber unterscheidet sich grundlegend von platonischen Positionen durch seine Haltung zum Kontingenzproblem. Weber will die Unbestimmtheit politischen Handelns nicht durch eine in letzten Wahrheiten gründende Herrschaftsstruktur ausschalten oder zumindest einschränken, sondern, ganz im Gegenteil, Kontingenz im Sinne eines Anders-Handeln-Könnens gegenüber den typisch modernen Zwängen der formalen Rationalisierung und Bürokratisierung erhalten ${ }^{74}$. Weber, und darin liegt die Besonderheit

71 Dieser Zusammenhang wird in geradezu groteskem Maße banalisiert, wenn Stefan Breuer Webers Eintreten für die charismatische Führerpersönlichkeit in den Diskussionen um die Weimarer Verfassung als bedauerliche Fehleinschätzung von Entwicklungstrends charakterisiert: »Weber, so scheint es, hat einfach Michels' Analysen über die quasi-militärische Hierarchie der deutschen Sozialdemokratie und die ihm zur Verfügung stehenden Informationen über die plebiszitäre Demokratie in Amerika addiert und zu einem Trend hochgerechnet, ohne dabei die - theoretisch von ihm durchaus erkannte - Möglichkeit einzubeziehen, dass die Demokratisierung auch zu einer Öffnung der hierarchischen Struktur, ja sogar zu ihrem Abbau führen kann" (Stefan Breuer, Bürokratie und Charisma, aaO. (FN 25), S. 173). Einen solchen Abbau hierarchischer Strukturen konnte Weber in seinem herrschaftskategorialen Verständnis von Politik gerade nicht wollen.

72 Dolf Sternberger, Drei Wurzeln der Politik, Bd. II, Frankfurt a.M. 1978, S. 355.

73 Edith Hanke zitiert hier Georg Simmel: Zur Philosophie der Herrschaft, in: ders., Soziologie. Untersuchungen über die Formen der Vergesellschaftung, Leipzig 1908, S. 197; zitiert nach Edith Hanke, »Max Webers >Herrschaftssoziologie<. Eine werkgeschichtliche Studie« in: Edith Hanke / Wolfgang Mommsen (Hg.), Max Webers Herrschaftssoziologie, Tübingen 2001, S. 25.

74 Zum Kontingenzproblem bei Weber vgl. Kari Palonen, Das»Webersche Moment«. Zur Kontingenz des Politischen, Wiesbaden 1998. 
seiner Position, hält nun gerade das von Sternberger mit Aristoteles als despotisch bezeichnete Verhältnis von Befehlen und Gehorchen, bzw. Entscheiden und Ausführen für das letzte Refugium der Freiheit. Bereits 1974 hat Wolfgang Mommsen darauf bezogen Webers Position in der paradoxen Formel »möglichst viel Freiheit durch möglichst viel Herrschaft« zusammengefasst ${ }^{75}$.

\section{Sinn-und Freibeitsverlust bei Hannah Arendt}

Auf den ersten Blick finden sich bei Weber und Arendt die selben Leitmotive der Kulturkritik: Bürokratisierung und Verantwortungslosigkeit, Funktionalisierung, Vermassung und Konformismus sowie die Flucht in Subjektivismus und blinden Genuss. Auch das parzellisierte, in den funktionierenden Fachmenschen und geistlosen Genussmenschen auseinanderfallende Individuum Webers und der sich nur noch verhaltende, in quasi automatischem Funktionieren und Konsumieren aufgehende und seine Individualität verlierende Animal laborans der Jobholder Society bei Arendt ${ }^{76}$ scheinen sich allenfalls in Nuancen zu unterscheiden. Ohne große interpretatorische Verrenkungen lässt sich deshalb Arendts Kritik der Moderne ebenfalls unter der Formel des Sinn- und Freiheitsverlustes zusammenfassen.

Die Nennung derselben Phänomene impliziert jedoch nicht unbedingt die Inkriminierung desselben Tatbestandes, derselben Gefahr oder Drohung. Zwar erzählen uns beide Autoren die Geschichte moderner Gesellschaften in dekadenztheoretischen Kategorien als Verlustgeschichte. Da sich ihr Ausgangspunkt unterscheidet, unterscheidet sich jedoch auch der jeweils beklagte Verlust. Max Weber misst die Moderne an der zu ethisch-methodischer Lebensführung fähigen Persönlichkeit, die im Ergebnis der von ihr eingeleiteten gesellschaftlichen Rationalisierungsprozesse zu verschwinden droht. Arendt dagegen bezieht die Maßstäbe ihrer Kritik aus der Möglichkeit des Handelns in pluralen öffentlichen Räumen, die sie in der antiken Polis und den politischen Revolutionen der Neuzeit verwirklicht sah.

Während Max Weber in der willkürlichen, aber wertbezogenen Zwecksetzung durch das Individuum bzw. den charismatischen Führer Rettung vorm Sinn- und Freiheitsverlust der Moderne sucht, bildet für Hannah Arendt im Gegensatz dazu die Verallgemeinerung von Zweck-Mittel-Beziehungen gerade das Grundübel neuzeitlicher Gesellschaften. Weber will formal rationalisierte Organisationen politisieren, indem er sie den wertrational bestimmten Zwecken starker Führer unterstellt. Für Arendt dagegen liegt zwischen Zweckrationalität und politischem Handeln der denkbar größte Widerspruch.

75 Wolfgang J.Mommsen, Max Weber. Gesellschaft, Politik, Geschichte, Frankfurt a.M. 1974, S. 138.

76 Hannah Arendt, Vita Activa oder vom tätigen Leben, München 1981, S. 314. 


\subsection{Sinnverlust durch Zweckrationalität}

Für Max Weber ist Sinn gleichbedeutend mit einem letzten, ursprünglich religiösen Zweck, auf den der einzelne Mensch seine Lebensführung ausrichten und rational gestalten kann. Bereits in den religionssoziologischen Grundlagen seiner Kulturkritik identifiziert Weber also Sinnstiftung mit der rationalen Beziehung auf einen Endzweck, der hier spezifischer als religiöse Heilsgewissheit bestimmt ist. Demgegenüber polemisiert Hannah Arendt gegen die weitverbreitete Gleichsetzung von Sinn und Zweck und versucht, die Bedeutung der beiden Begriffe grundsätzlich zu unterscheiden.

Ihr zufolge gründen die Pathologien der Moderne nicht zuletzt in der Unfähigkeit des Homo faber, den Unterschied zwischen dem Nutzen und dem Sinn einer Sache zu verstehen. Ein Tun im Modus des »Um-zu«, d.h. um einen bestimmten Zweck zu erreichen, gerät, wie Arendt argumentiert, unweigerlich in einen $Z_{\text {weck- }}$ progressus ad infinitum«. Denn ohne Überwindung des Nützlichkeitsdenkens bestehe keine Möglichkeit, die bereits von Lessing gestellte Frage: »Und was ist der Nutzen des Nutzens? « zu beantworten ${ }^{77}$. Stärker noch: da der Homo faber der Neuzeit das Nützlichkeitsdenken über den Herstellungsprozess hinaus verallgemeinere, verursache er

»die Degradierung aller Welt- und Naturdinge zu bloßen Mitteln, die unaufhaltsame Entwertung alles Vorhandenen, das Anwachsen der Sinnlosigkeit, in dessen Prozeß alle Zwecke verschlungen werden, um wieder zu Mitteln zu werden... « ${ }^{78}$.

In der Tat kann ohne einen feststehenden Endzweck, wie ihn der religiöse Mensch noch im Erlangen des ewigen Lebens hatte, jeder Zweck wiederum selbst zum Mittel für weitere Zwecke werden. Damit erweist sich zweckrationales Denken als unfähig, Sinn zu erzeugen, oder anders: »wo der Nutzen sich als Sinn etabliert, (wird) Sinnlosigkeit erzeugt « ${ }^{79}$.

Zweckrationales Handeln ist für Arendt also nicht das Gegenmodell zum Funktionalismus moderner Gesellschaften, sondern bringt ihn hervor. Damit ist impliziert, dass Arendts Sinnbegriff eine Qualität jenseits der Struktur der Zweckrationalität aufweisen muss. Es fragt sich nur, woher sie kommen und worin sie bestehen soll?

Zunächst setzt auch Arendt am modernen Glaubensverlust an und betont, dass er nicht nur die Gewissheit eines jenseitigen Lebens betrifft, sondern auch die diesseitige Welt in Frage stellt. Dabei denkt sie jedoch nicht nur an das Problem der beliebig gewordenen Wahl zwischen konfligierenden Werten, das Weber mit der Metapher des neuen Polytheismus immer wieder anspricht. Die Schrecken totaler Herrschaft hatten ihr ganz andere Konsequenzen des Verfalls letzter Werte dramatisch vor Augen geführt. In einem Interview formuliert sie 1972: »I am perfectly sure that this whole totalitarian catastrophe would not have happened if people still believed in God, or hell rather - that is if there were still ultimates « ${ }^{80}$.

77 Ebd., S. 141.

78 Ebd., S. $143 \mathrm{f}$.

79 Ebd., S. 141.

80 "Hannah Arendt on Hannah Arendt« (AoA), in: Melvyn Hill (ed.), Hannab Arendt. The Recovery of the Public World, New York 1979, S. $313 \mathrm{f}$. 
Arendt wie Weber waren von Dostojewski und seiner religiösen Kritik der Moderne beeinflusst. Die Formel, mit der Hannah Arendt das Selbstverständnis totaler Herrschaft auf den Punkt bringt, lautet: »Alles ist möglich«, gewissermaßen eine Steigerung von Dostojewskis »Alles ist erlaubt «. Ähnlich wie Weber hält allerdings auch Arendt daran fest, dass es allgemeinverbindliche letzte Werte nicht mehr gibt und sie durch Rückbesinnung auf Tradition oder Religion auch nicht wieder zu beleben sind. Der Mensch der Neuzeit ist auf sich zurückgeworfen und muss seinem Leben selbst einen Sinn geben. Sinn entsteht für sie aber nicht aus der Willensentscheidung des einsamen Individuums oder des charismatischen Führers, sondern nur im Zusammenwirken mit Anderen in einer interpersonal geteilten Welt. Genauer entsteht Sinn durch das Handeln mit und vor Anderen, die als Gleiche untereinander verkehren und ihre Angelegenheiten gemeinsam regeln. Während für Weber die Sinnhaftigkeit eines Tuns aus einer vorpolitischen und rational unbegründbaren Entscheidung für bestimmte Werte folgt, besitzt für Arendt das politische Handeln selbst die Fähigkeit, Sinn zu erzeugen.

\subsection{Neubeginn, Pluralität und Weltlichkeit als Dimensionen des Handelns}

Das ist nun allerdings erläuterungsbedürftig. Die Antwort auf die Frage, weshalb gerade die Politik in der Lage sein sollte Sinnfragen zu lösen, liegt in Arendts emphatischem Begriff des Handelns. Handeln ist für Arendt neben dem Arbeiten und Herstellen eine der drei Grundtätigkeiten des tätigen Lebens. Grob vereinfacht lässt sich Arbeiten als funktional, durch naturhafte Notwendigkeit bestimmt, Herstellen als instrumental und Handeln als interpersonal charakterisieren. Handeln ist zunächst einmal identisch mit dem Beginnen von etwas Neuem. Augustinus zitierend, »damit ein Anfang sei wurde der Mensch geschaffen«, behauptet Arendt, der Mensch könne, »weil er ein Anfang und Neuankömmling in der Welt sei«, auch Initiative ergreifen und Neues in Bewegung setzen ${ }^{81}$. Durch diese Fähigkeit des Neuanfangens, durch seine Spontaneität kann der Mensch aus den quasi naturhaften Lebensprozessen der Gesellschaft heraustreten und sich »aller Absehbarkeit und Berechenbarkeit « entziehen $^{82}$. Handeln bildet die Alternative zum bloßen Sich-Verhalten, zum reibungslosen und automatischen Funktionieren der Arbeitsgesellschaft.

Anders als bei Weber haben wir es bei Arendts (fast idealtypischer) Unterscheidung von Tätigkeiten nicht mit einer sozialwissenschaftlichen Theorie gesellschaftlicher Rationalisierung zu tun, sondern mit einer existentialphilosophischen Bestimmung menschlicher Bedingungen und Möglichkeiten. Arendt weist zwar explizit zurück, eine Anthropologie zu entwickeln, oder Aussagen über die »Natur« des Menschen zu machen, weil ihr zufolge ein solches Vorhaben der prinzipiel-

81 Hannah Arendt, Vita Activa, aaO. (FN 76), S. 166.

82 Ebd., S. 167.

ZfP 52. Jg. 1/2005 
len Offenheit menschlicher Existenz widerspräches3. Dennoch bleibt festzuhalten, dass sie ihre Gesellschaftskritik aus existentialphilosophisch begründeten, normativen Aussagen über Möglichkeiten des menschlichen Lebens entwickelt. Der Unterschied zur Rationalisierungstheorie Max Webers relativiert sich jedoch, wenn wir uns erinnern, dass seine Kritik an der Durchsetzung formaler Rationalität ebenfalls auf normative, allerdings philosophisch nicht weiter begründete Vorstellungen von »Menschentum « und selbstbestimmtem Handeln rekurriert, die ihren Ursprung im deutschen Idealismus schwerlich verbergen können.

Während Weber davon ausgehend eine freiheitssichernde Politik am Typ zweckrationalen Handelns orientiert und sich damit die Affirmation direkter Herrschaft einhandelt, gilt es nun zu klären, ob Arendt mit ihrer existentialistischen Bestimmung menschlicher Bedingungen und Möglichkeiten einen tragfähigen Grund für eine sinnstiftende und freiheitsverwirklichende politische Praxis gewinnen kann.

Die bereits erwähnte Fähigkeit des Handelns zum Neubeginn allein reicht dazu sicher nicht aus. Auch in anderen Tätigkeiten, etwa in der Produktivität des künstlerischen Herstellens, gibt es nach Arendt ein Moment der Spontaneität ${ }^{84}$, ohne dass diese dadurch bereits als politische Tätigkeiten qualifiziert wären. Die weiteren Dimensionen des Handelns, die erst zusammen seine politische Qualität ausmachen, sind Pluralität, Sprachlichkeit und Weltlichkeit.

Das Faktum der Pluralität ist nach Arendt Grundbedingung des politischen Handelns nicht nur im Sinne einer »conditio sine qua non«, sondern auch als »conditio per quam $\ll^{85}$. Denn nur indem wir vor und mit anderen handeln, kann das Unterschiedensein jeder Person hervortreten und wirklich werden ${ }^{86}$. Wir handeln nicht nur weil wir verschieden sind, sondern auch damit wir unsere Verschiedenheit realisieren können.

Sprechen und Handeln gehören bei Arendt aufs Engste zusammen. Sie unterscheidet beides zwar insofern, als sie dem Handeln eher die Dimension des Beginnens, dem Sprechen die der Selbstenthüllung des Handelnden zuordnet. Streng genommen aber gibt es für sie ein Handeln ohne Sprechen gar nicht, und zwar zum einen deswegen, weil es ein Handeln ohne Handelnden wäre, ihm also die revelatorische Dimension des »Wer« der Tat fehlte, zum anderen aber, weil Handeln ohne sprachliche Kommunikation mit anderen sinnlos bleiben müsste und von zweckra-

83 Vgl. Hannah Arendt, Vita Activa, aaO. (FN 76), S. 16 und S. 18. Die Frage, ob es sich bei Arendts Bestimmung der Grundtätigkeiten um eine Anthropologie handelt oder nicht, ist in der Sekundärliteratur umstritten. Benhabib spricht vom "anthropologischen Universalismus « Arendts, Estrada Saavedra dagegen widerspricht dem unter Hinweis auf Arendts eigene Position sowie ihrer Ablehnung aller Aussagen über den Menschen als »Gattung " (Vgl. Seyla Benhabib, The Reluctant Modernism, aaO. (FN 4) S. 195; Marco Estrada Saavedra, Die deliberative Rationalität des Politischen, Würzburg 2002, S. 22).

84 Hannah Arendt, Was ist Politik? (WiP) Fragmente aus dem Nachlaß, München 1993, S. 51.

85 Hannah Arendt, Vita Activa, aaO. (FN 76), S. 15.

86 Ebd., S. 164. 
tionaler Tätigkeit oder Gewalt nicht zu unterscheiden wäre. »Erst durch das gesprochene Wort fügt sich die Tat in einen Bedeutungszusammenhang. «87.

Sinnhaftes Handeln ist deswegen an ein Miteinander des Sprechens und Agierens gebunden, das Arendt sowohl vom reinen »Gegeneinander« als auch von einem selbstlosen »Füreinander « abgrenzt ${ }^{88}$. Handeln bedarf der Referenz auf Andere. Es bedarf einerseits eines sprachlich vermittelten Sinnhorizontes, konstituiert andererseits jedoch selbst das "Gewebe menschlicher Bezüge und Angelegenheiten $\ll^{89}$, in dem es erst Bedeutung gewinnen kann.

In diesen Zusammenhang gehört auch die bisweilen befremdliche Faszination Arendts vom »agonalen Geist « der Griechen und deren Streben nach Ruhm und Unsterblichkeit. Arendt sieht im politischen Raum einen Raum des Wettstreits, der es den Individuen ermöglicht, in Erscheinung zu treten und sich vor anderen auszuzeichnen. Das »Bezugssystem zwischen den Menschen, das aus Handeln und Sprechen entsteht«, ist zwar weniger handgreiflich und fluider als die vom Homo faber geschaffenen Gegenstände, aber in ihm können die Menschen »...Dinge tun und Worte sprechen, ...die der Unsterblichkeit würdig, also wert sind, für immer erinnert zu werden $\ll^{90}$. Sehen wir vom elitären Aspekt dieses Gedankens ab, so bleibt, dass Handeln einen pluralistisch geprägten »öffentlichen Erinnerungsraum «, einen »kommunikativen Ressonanzboden ${ }^{91}$ hervorbringt, der Narration und Sinnstiftung ermöglicht.

Sinn entsteht bei Arendt deshalb nicht aus der einsamen Entscheidung über ein zu verfolgendes Ziel, sondern in der Perspektivenpluralität einer mit anderen geteilten Welt. Umgekehrt folgt Sinnverlust dann nicht aus der Pluralisierung von Werten, sondern aus der Zerstörung dieser pluralen, aber gemeinsamen Welt durch Herrschaft und Funktionalisierung. Dabei bedeutet der Funktionalismus der mo-

87 Ebd., S. 168.

88 Ebd., S. 169. Damit ist auch eine Begrenzung der während der letzten Jahre geführten Debatten um eine agonale oder kommunikative Interpretation des Handelns bei Arendt markiert. Das Erscheinen vor Anderen bleibt immer an einen gemeinsamen Sinnhorizont rückgebunden und kann deshalb trotz des vorhandenen Elements von Wettstreit kaum im Sinne Nietzsches als agonal verstanden werden. $\mathrm{Zu}$ einer eher agonalen Interpretation Arendts, s. Bonnie Honig, Political Theory and the Displacement of Politics, Ithaca/London 1993; Dana Villa, Arendt and Heidegger, aaO. (FN 61); Zur Kritik an diesen Interpretationen etwa Lawrence J. Biskowski, »Politics versus Aesthetics: Arendt's Critiques of Nietzsche and Heidegger « in: The Review of Politics, Vol. 57, 1/ 1995 und Seyla Benhabib, The Reluctant Modernism, aaO. (FN 4).

89 Hannah Arendt, Vita Activa, aaO. (FN 76), S. 87.

90 Hannah Arendt, »Kultur und Politik « (KP) in: Zwischen Vergangenheit und Zukunft, München 1994, S. 287.

91 Frank Nullmeier, »Agonalität - Von einem kultur- zu einem politikwissenschaftlichen Grundbegriff? « in: Michael Th. Greven/Herfried Münkler/Rainer Schmalz-Bruns (Hg.), Bürgersinn und Kritik. Festschrift für Udo Bermbach zum 60. Geburtstag, Baden-Baden 1998, S. 101. Nullmeier interpretiert das agonale Moment in Arendts Politikbegriff überzeugend in diesem Sinn und grenzt es damit implizit vom potentiell gewaltsamen Kampf bei Weber ab. 
dernen Arbeitsgesellschaft gegenüber der Zweckrationalität traditioneller Herrschaft für Arendt eine neue Qualität in der Zerstörung von Sinn und Freiheit. In ihrer Auseinandersetzung mit Marx übernimmt sie dessen Grundgedanken der Verselbständigung ökonomischer Verhältnisse. Sie kritisiert die funktionale Integration der Individuen in modernen Wirtschaftsgesellschaften als eine freiheitszerstörende Naturalisierung, die interpersonale Beziehungen dem Diktat der vermeintlichen Notwendigkeiten des gesellschaftlichen Lebensprozesses unterstellt. In dieser Hinsicht sind Weber wie Arendt durch die Marxsche Kapitalismuskritik geprägt ${ }^{22}$. Die Parallelen der Kritik verselbständigter Handlungszusammenhänge bei Weber und Arendt sind offensichtlich in Arendts Bezeichnung der Bürokratie als »Niemandsherrschaft «, die die tyrannischte Staatsform überhaupt sei, weil in ihr keine Person oder Gruppe mehr für irgend etwas verantwortlich gemacht werden kann ${ }^{93}$.

Ausgehend von ihrer Unterscheidung zwischen den drei Grundtätigkeiten des Handelns, Herstellens und Arbeitens versteht Arendt die moderne Wirtschaftsgesellschaft als einen durch natürliche Notwendigkeiten bestimmten oikos im erweiterten Maßstab der Nation ${ }^{94}$. Die Auslieferung der modernen Menschen an den funktional bestimmten, naturhaften Reproduktionsprozess der Gesellschaft zerstört sowohl die Pluralität ihres »Bezugsgewebes« als auch die Stabilität und Verlässlichkeit ihrer gemeinsamen Welt.

\subsection{Arendts existentialphilosophischer Weltbegriff als Grundlage ibres Bruches mit dem herrschaftszentrierten Politikverständnis}

Bei Weber ist die Welt stets Chiffre für die dem Subjekt entgegengesetzten Zwänge und Eigengesetzlichkeiten, wie sie letztlich aus dem, wie er formuliert, »ewigen Kampf des Menschen mit dem Menschen auf der Erde «95 resultieren und an denen sich jeder Versuch der ethischen Rationalisierung brechen muss ${ }^{96}$. Bei Arendt bezeichnet der Begriff der Welt ein »Zwischen«, ein Beziehungsgeflecht, das sinnhaftes Handeln erst ermöglicht.

92 Arendt wirft Marx vor, mit seinem Begriff des »Gattungswesens « diese Naturalisierung zum kollektiven Lebensprozess einer vergesellschafteten Menschheit befördert zu haben. Dabei lässt sie völlig außer acht, dass Marx den Charakter einer »zweiten Natur «, den ihm zufolge die kapitalistische Warenwirtschaft angenommen hat, in der Zweckrationalität einer geplanten Ökonomie auflösen wollte. Zu Arendts Fehlinterpretation der Marxschen Verdinglichungskritik vgl. Bikhu Parekh, "Hannah Arendts Critique of Marx in: Melvyn Hill (ed.), The Recovery of the Public World, New York 1979, S. 67-100.

93 Hannah Arendt $M G$, aaO. (FN 62), S. $39 f$.

94 Vgl. Hannah Arendt, Vita Activa, aaO. (FN 76), S. 31, S. 105.

95 Max Weber, GPS, aaO. (FN 35), S. 29.

96 Webers Weltbegriff wird besonders deutlich in der "Zwischenbetrachtung (ZB 552f.) und in der bekannten Diskussion des Verhältnisses von Gesinnungs- und Verantwortungsethik (GPS 547ff.) 
Damit sind wir bei dem vielleicht grundlegendsten Unterschied zwischen den Politikbegriffen von Arendt und Weber angelangt. Ernst Vollrath hat darauf hingewiesen, dass sich im Weltverständnis Webers nicht nur ein »nietzscheanisches Willens-, Macht- und Kampfmotiv« reflektiere, sondern grundsätzlicher noch die im deutschen Kulturraum dominierende realpolitische, herrschaftskategorial bestimmte Apperzeption des Politischen. Aus ihr folge ein komplementär-antagonistisches Verhältnis von realistischer Macht- und ethischer Idealpolitik, in dessen Bezugsrahmen eine zivilpolitische, auf Zustimmung, Assoziation und differentielle Einheit bezogene Qualität des Politischen nicht zu denken sei ${ }^{97}$. Es ist unschwer zu erkennen, wie sich ein entsprechend komplementär-antagonistisches Verhältnis auch in Webers berühmter Gegenüberstellung von Gesinnungs- und Verantwortungsethik ${ }^{98}$ und, auf unser Thema bezogen, im Verhältnis zwischen der freien Willensentscheidung des Individuums für letzte Werte und den Sachgesetzlichkeiten der Welt wiederfindet.

Arendt verortet den Ursprung der herrschaftskategorialen Wahrnehmung des Politischen weit hinter irgendwelchen Besonderheiten der deutschen Geschichte in den Anfängen der abendländischen Philosophie. Bereits das Denken Platons habe das plurale politische Handeln der griechischen Polis nach dem Vorbild des Herstellens transformiert und in Befehlen und Gehorchen aufgelöst ${ }^{99}$. Wie zuvor nur im Verhältnis zwischen Herr und Sklave können damit dann auch im politischen Bereich Gewalt sowie Befehls-Gehorsamsbeziehungen durch Zweckrationalität und Herrschaftswissen gerechtfertigt werden. Offenkundig gründet für Arendt der Herrschaftsbegriff des politischen Denkens in technischer Rationalität und der ihr entsprechenden Teilung von Wissen und Tun ${ }^{100}$.

Arendt scheint damit lediglich eine Variante der bekannten neoaristotelischen Kritik an einem herrschafts- statt bürgerschaftszentrierten Begriff des Politischen zu vertreten. Die Originalität ihres Denkens, insbesondere aber der von ihr hergestellte Zusammenhang von Kulturkritik und Demokratie erschließt sich jedoch, sobald wir ihre Uminterpretation des Heideggerschen Weltbegriffes berücksichtigen, durch die sie den Bruch mit einer herrschaftskategorialen Wahrnehmung des Politischen vollzieht. Zunächst einmal bezeichnet der Begriff der »Welt« bei Arendt nicht eine dem Willen des Subjektes und seinen Werten entgegengesetzte Wirklichkeit, sondern ein "Zwischen «, das freiheitliches Handeln erst ermöglicht. Mit diesem "Zwischen« meint sie zweierlei: zum einen die gegenständliche Welt, auf die sich Menschen aus verschiedener Perspektive handelnd beziehen, zum anderen den Erscheinungsraum, in dem sich Menschen aneinander richten und ein »Bezugsgewebe

97 Vgl. dazu auch Ernst Vollrath, »Max Weber«, aaO. (FN 58), S. 103.

98 Vgl. dazu ausführlich und kenntnisreich Lothar Waas, Max Weber und die Folgen. Die Krise der Moderne und der moralisch-politische Dualismus des 20. Jabrhunderts, Frankfurt a.M. 1995.

99 Vgl. Hannah Arendt, Vita Activa, aaO. (FN 76), S. 219.

100 Mit explizitem Hinweis auf Heidegger formuliert Arendt dies auch in den "Denktagebüchern« in einer Interpretation des Gerechtigkeitsdialogs in Platons Politeia (Hannah Arendt, Denktagebuch, 1950-1973, 2 Bde, München 2002, S. 206). 
menschlicher Angelegenheiten «101 bilden. Beide Aspekte des Weltbegriffes bedingen sich gegenseitig. Während der Erscheinungsraum und ein Minimum des Vertrauens in Sprechen und Handeln als Weisen des Miteinander für uns erst Wirklichkeit konstituieren, kann umgekehrt der Erscheinungsraum überhaupt erst entstehen durch die verschiedenen Bezüge der Vielen auf ein ihnen gemeinsam Entgegenstehendes. Arendt vergleicht diese Funktion der gegenständlichen Welt mit einem Tisch, der diejenigen, die um ihn herumsitzen, zugleich verbindet und trennt ${ }^{102}$. Wo die Welt diese Fähigkeit zu versammeln, das heißt zu verbinden und zu trennen zugleich, verliert, kommt es dazu, dass die Menschen atomisiert werden oder in eins fallen. Beide Phänomene kennzeichnen die moderne Massengesellschaft und bilden nach Arendt die Voraussetzung für den Erfolg totalitärer Bewegungen ${ }^{103}$.

Mit Heideggers Begriff der Welt übernimmt Arendt auch dessen Kritik an der Epistemologie der modernen Wissenschaft, insbesondere an der mit Descartes identifizierten Trennung zwischen Subjekt und Objekt der Erkenntnis. Die »Welt« ist nicht der Gegenstand eines erkennenden oder wollenden Subjektes, sondern ein Bezugsgewebe, das stets Um-welt und Mit-welt zugleich ist ${ }^{104}$. Für Arendt wie für Heidegger ist deshalb das »In-der-Welt-Sein « immer schon ein »Mitsein mit Anderen«. Während jedoch Heidegger im Mitsein eine inauthentische Form des Daseins sieht, eine Verfallenheit an das »Man«, wertet Arendt den Weltbegriff so um, dass das Mitsein mit Anderen sowohl zur Möglichkeitsbedingung als auch zum immanenten Ziel politischen Handelns wird. Der Begriff einer interpersonal konstituierten Welt, wie ihn Arendt aus den existentialphilosophischen Einflüssen von Heidegger und Jaspers entwickelt ${ }^{105}$, eröffnet ihr einen Weg aus der Zweckrationalität von Subjekt-Objekt-Beziehungen und damit aus den Aporien der Weberschen Rationalisierungstheorie. Er ermöglicht es, politisches Handeln als diesseitige Erzeugung von Sinn und - wie gleich zu begründen sein wird - als Verwirklichung von Freiheit zu verstehen. Mit anderen Worten, Arendt kann Politik als Praxis unter Gleichen denken.

101 Hannah Arendt, Vita Activa, aaO. (FN 76), S. 173.

102 Ebd., S. 52.

103 Der Analyse der modernen Weltentfremdung kommt bereits in Arendts erstem Hauptwerk, in »Elemente und Ursprüngen totaler Herrschaft « zentrale Bedeutung zu (vgl. Hannah Arendt, Elemente und Ursprünge totaler Herrschaft, München 1986 (1958)). Ausführlicher zur Weltentfremdung bei Arendt Winfried Thaa, »Hannah Arendt. Politik und Weltentfremdung «, in: Politische Vierteljabresschrift, 38 Jg., 4/1997, S. 695-715.

104 Der Weltbegriff bei Arendt und seine Wurzeln in der Philosophie Heideggers sind während der letzten Jahre verstärkt in den Mittelpunkt der Arendtforschung gerückt. Dazu etwa: Dana R. Villa, aaO. (FN 61), insbes. S. 117-129; Rahel Jaeggi, Welt und Person: Zum anthropologischen Hintergrund der Gesellschaftskritik Hannab Arendts, Berlin 1997; Seyla Benhabib, The Reluctant Modernism, aaO. (FN 4) sowie Janita Hämäläinen, »Arendt und Heidegger - Konvergenz in der > Welt $»$ in: Hannab Arendt Newsletter, 3/2000, S. 18-23.

$105 \mathrm{Zu}$ Jaspers Einfluss auf Arendt vgl. Lewis P. Hinchman / Sandra K. Hinchman, »Existentialism Politicized. Arendt's Debt to Jaspers « in: Lewis P. Hinchman / Sandra K. Hinchman (eds.), Hannah Arendt. Critical Essays, Albany 1994, S. 143-178. 


\subsection{Freibeit als nichtsouveränes Handeln}

Entsprechend versteht Hannah Arendt Freiheit als ein politisches Phänomen, »das primär weder im Wollen noch im Denken, sondern im Handeln erfahren wird «106. Dabei geht sie von Montesquieus Unterscheidung zwischen philosophischer und politischer Freiheit aus, wonach die erste eine Freiheit des Willens bezeichne, die zweite eine des Könnens innerhalb gesetzlich begrenzter Möglichkeiten ${ }^{107}$.

»Die politische Freiheit unterscheidet sich also von der philosophischen Freiheit dadurch, daß sie eindeutig eine Sache des Ich-kann und nicht des Ich-will ist. Da sie dem Bürger und nicht dem Menschen überhaupt zukommt, kann sie sich nur in Gemeinschaften zeigen, wo die vielen Zusammenlebenden in Wort und Tat miteinander verkehren, geregelt durch viele rapports - Gesetze, Sitten, Gebräuche und Ähnliches. Mit anderen Worten, die politische Freiheit ist nur möglich in der Sphäre der menschlichen Pluralität ...«108.

Gegen die philosophische und christliche Tradition, aber auch gegen moderne Vorstellungen betont Arendt immer wieder, politische Freiheit sei nicht als Willensfreiheit oder Souveränität, das heißt als ein Phänomen des Selbstbezuges im Sinne von »Ich tu, was ich will« zu verstehen. Vielmehr sei Freiheit ein Phänomen des Verkehrs mit anderen ${ }^{109}$. Der Singularität des Ich-will entspringe die Tyrannis ${ }^{110}$, und die Souveränität, die säkularisierte Idee göttlicher Allmacht, stehe in diametralem Gegensatz zur politischen Freiheit, weil »Souveränität, nämlich unbedingte Autonomie und Herrschaft über sich selbst, der menschlichen Bedingtheit der Pluralität widerspricht« ${ }^{111}$.

"Wie die Souveränität des einzelnen ist letztlich auch die Souveränität einer Gruppe oder eines politischen Körpers immer nur Schein; sie kann nur dadurch zustande kommen, daß eine Vielheit sich so verhält, als ob sie einer wäre und dazu noch ein einziger [...]. Wo Menschen, sei es als einzelne, sei es in organisierten Gruppen, souverän sein wollen, müssen sie die Freiheit abschaffen. Wollen sie aber frei sein, so müssen sie auf Souveränität geradezu verzichten « ${ }^{112}$.

106 Hannah Arendt, »Freiheit und Politik« (FP) in: Zwischen Vergangenheit und Zukunft, München 1994, S. 210.

107 Hannah Arendt, Über die Revolution ( $\ddot{U} R)$, München $1986^{3}$ (1965), S. 380, und ähnlich Hannah Arendt, Vom Leben des Geistes (LG), München 1998, S. 425

108 Ebd., S. 426.

109 In diesem Sinn etwa Hannah Arendt, FP, aaO., (FN 106), S. 201, 210ff; $\ddot{U} R$ aaO. (FN 107), S. 194; WiP, aaO. (FN 84), S. 38ff.).

110 Hannah Arendt, FP, aaO. (FN 106), S. 213.

111 Vgl. dazu u.a. Hannah Arendt, Vita Activa, aaO. (FN 76), S. 229. Auch hier liegt der Argumentation Arendts eine existentialistisch geprägte Kritik an der Identitätsphilosophie zugrunde. Sofern Freiheit vom Ideal der Selbstidentität aus gedacht wird, kann sie die Welt als Bedingung allen Handelns und damit auch Pluralität nur als Schranke wahrnehmen. Zu diesem Zusammenhang ausführlicher Ernst Vollrath, »Ein philosophischer Begriff des Politischen? « in: Neue Hefte für Philosophie, 21. Jg., 1/1982.

112 Vgl. Hannah Arendt, FP, aaO. (FN 106), S. 215. 
Freiheit, die als Willensfreiheit gedacht wird, ist letztlich die Fähigkeit, die eigenen Ziele gegen andere durchzusetzen. Das aber ist nicht nur antipluralistisch und für Arendt damit antipolitisch, es verweist zugleich auf Gewalt als das wirkungsvollste Mittel, andere zu etwas zu zwingen, was sie nicht wollen.

\subsection{Die amerikanische Revolution}

Erst von dieser, im existentialphilosophischen Begriff der Welt gründenden Kritik der Gleichsetzung von Freiheit und Souveränität erschließt sich Arendts Interpretation der amerikanischen Revolution bzw. ihre für eine deutsche Emigrantin so erstaunliche »Entdeckung der Freiheit «113 in der amerikanischen Demokratie. Für Arendt gelang es der amerikanischen Revolution, »den Anspruch der Macht auf Souveränität im politischen Körper der Republik konsequent zu eliminieren «114 sowie in Verfassung und politischen Institutionen einen pluralen öffentlichen Raum zu schaffen, in dem Menschen handeln und ihre Macht im Rahmen von Regeln und Gesetzen ausüben konnten. Nach Arendt bestand bereits vor der Revolution die Einzigartigkeit der amerikanischen Politik darin, dass die Siedler sich als »civil Bodies Politick « oder "politische Bürgerschaften « zusammenschlossen, in denen es keine Herrscher und Beherrschte gab, die vielmehr einen politischen Raum bildeten, »in dem Macht und die Beanspruchung von Rechten möglich war, ohne dass man doch Souveränität besaß oder auch nur nach ihr verlangte «115. Auf dem Hintergrund dieser Erfahrung hätten die Gründungsväter, und hierbei insbesondere Madison, Montesquieus Lehre von der Gewaltenteilung neu interpretiert und eine föderative republikanische Staatsform entwickelt, die als Zusammenschluss oder »cosociation« verschiedener politischer Handlungsräume zu verstehen sei ${ }^{116}$.

Während Max Weber, wie wir vorne gesehen haben, diese bürgerschaftlichen Formen der Politik dazu verurteilt sah, dem doppelten Druck der unvermeidlichen Rationalisierung und Bürokratisierung auf der einen, sowie dem Element des »Kampfes« in der großen Politik auf der anderen Seite zu weichen, erkannte Arendt in ihnen eine neuzeitliche, pluralistische Form des Republikanismus, die nicht nur eine Alternative zur Herrschaftslogik des abendländischen politischen Denkens bot, sondern ihr darüber hinaus geeignet schien, den Zerfall verbindlicher Werte in modernen Gesellschaften politisch, das heißt durch plurales Handeln unter Gleichen, statt durch dezisionistische Willkür und Befehls-Gehorsamsbeziehungen zu bewältigen. Bei aller Kritik an der amerikanischen Politik ihrer Zeit und dem Konsumismus der amerikanischen Gesellschaft, war Arendt doch überzeugt davon, in den Institutionen der Republik und dem politischen Geist des amerikanischen Gemeinwesens das entscheidende Gegenprinzip zum Sinn- und Freiheitsverlust der

113 Vgl. dazu Winfried Thaa / Lothar Probst (Hg.), Die Entdeckung der Freibeit. Amerika im Denken Hannab Arendts, Berlin 2003.

114 Vgl. Hannah Arendt, $\ddot{U} R$, aaO. (FN 107), S. 200.

115 Ebd.

116 Ebd. 
Moderne gefunden zu haben: nämlich »die dauerhafte Teilnahme an allen Angelegenheiten von öffentlichem Belang «117. Diese Perspektive politischer Partizipation richtet sich bei Arendt allerdings nicht auf die möglichst unverfälschte Durchsetzung eines wie immer gearteten Volkswillens, sondern auf die Möglichkeiten der Bürger, sich im Austausch mit anderen eine Meinung zu bilden und an der Gestaltung ihrer gemeinsamen Angelegenheiten teilzunehmen. Durch das Eindringen vermeintlicher Notwendigkeiten in den öffentlichen Raum, genauer durch die Naturalisierung der Ökonomie zum gesellschaftlichen Lebensprozess werden diese Handlungsmöglichkeiten untergraben.

Ein entscheidendes Versäumnis der amerikanischen Revolution liegt Arendt zufolge darin, dass sie die Möglichkeiten zur direkten Bürgerbeteiligung nicht in dem $\mathrm{Maße}$, wie es Jefferson gefordert hatte, institutionalisierte ${ }^{118}$. In den dennoch vorhandenen Formen der Selbstverwaltung und Beteiligung, vor allem aber in den mit der Bürgerrechts- und Studentenbewegung der sechziger Jahre wieder stärker hervortretenden informellen Zusammenschlüssen und Vereinigungen konnte sie jedoch spezifisch amerikanische Phänomene sehen, die es dem Einzelnen zumindest zeitweise erlaubten, in öffentlichen Räumen zu handeln und damit sowohl Gemeinsinn wie Urteilsfähigkeit auszubilden ${ }^{119}$. Mit Bezug auf die amerikanische Regierung stellte Arendt allerdings schon vor dreißig Jahren fest, dass sie nicht mehr im Sinne der Gründungsväter, sondern im Sinne des europäischen nationalstaatlichen Denkens und dessen Souveränitätsbegriff handle. Wenn wir an die jüngsten Konflikte zwischen Europa und den USA denken, lässt sich darüber hinaus behaupten, die Fronten hätten sich regelrecht umgekehrt: Ein auf Gewalt gegründetes Politikverständnis und das Prinzip nationalstaatlicher Souveränität werden heute von den USA vertreten (sofern es um die eigene Souveränität geht), die Begrenzung nationaler Souveränität durch das internationale Recht dagegen von Europa.

\section{Autonome Dezision oder erweiterte Denkungsart}

Der Gegensatz zwischen Max Weber und Hannah Arendt tritt nirgendwo klarer hervor, als in den jeweiligen Lösungen, mit denen sie dem Verfall letzter Werte in modernen Gesellschaften begegnen wollen. Zunächst einmal stimmen jedoch beide darin überein, dass die Politik eine gesicherte normative Grundlage in Religion oder Metaphysik verloren hat und uns kein Rückweg zu den unstrittigen Werten der Tradition offen steht. Insofern stellen sie sich der ernüchternden Einsicht, wonach die Moderne »ihre Normativität aus sich selbst schöpfen (muß) «120. Die Frage, wie dies geschehen und nach welchen Kriterien Politik beurteilt werden kann, erhält für beide eine besondere Dringlichkeit daraus, dass sie politische Handlungsmöglichkeiten

117 Hannah Arendt, "Ziviler Ungehorsam« in: Zur Zeit. Politische Essays, München 1989, S.144.

118 Vgl. Hannah Arendt, ÜR, aaO. (FN 107), S. $319 f$.

119 Vgl. Hannah Arendt, "Ziviler Ungehorsam«, aaO. (FN 117), S. $155 f$.

120 Jürgen Habermas >Der philosophische Diskurs der Moderne, Frankfurt a.M. 1988, S. 16. 
gegen die Versachlichungs- und Funktionalisierungstendenzen moderner Gesellschaften erhalten bzw. zurückgewinnen wollen.

Webers Lösung, die willkürliche Entscheidung des Individuums bzw. des charismatischen Führers zwischen letzten Werten, schließt in Verbindung mit seiner Ablehnung jedes Kompromisses oder Mittelwegs im Konflikt von Wertordnungen eine wie auch immer geartete Rationalisierung ethischer Entscheidungen im politischen Raum aus. Webers wiederholte Appelle zur ehrlichen und rückhaltlosen Selbsterforschung der eigenen Wertaxiome relativieren die radikale Individualisierung und $\mathrm{Be}-$ liebigkeit der Entscheidung keineswegs. Ebenso wenig kann die nüchterne Kalkulation möglicher Handlungsfolgen, die Weber von der empirischen Wissenschaft erhoffte, normative Kriterien zu ihrer Beurteilung liefern. Das »Augenmaß«, das Weber vom Politiker fordert ${ }^{121}$ als »Urteilskraft« oder »praktische Klugheit« zu interpretieren ${ }^{122}$, täuscht über diese Schranke hinweg. Denn im Unterschied zur vormodernen praktischen Philosophie fehlen Webers Politiker die Maßstäbe des Urteils. In »Politik als Beruf « findet sich eine Anspielung auf Martin Luther: Mit einem »Ich kann nicht anders, hier stehe ich «, kennzeichnet Weber die letzte Wertbindung des verantwortlichen Politikers ${ }^{123}$. Der Protestant Webers ist allerdings eine höchst dubiose Gestalt, kam ihm doch schon lange die Heilige Schrift abhanden, mit der er seine Gewissensentscheidung vor sich und anderen begründen könnte.

Im Gegensatz dazu lässt sich Arendts Werk als Versuch lesen, im »Denken ohne Geländer «, wie sie es wiederholt formuliert ${ }^{124}$, einen Weg zwischen dem Abgrund dezisionistischer Willkür und der freiheitszerstörenden Suche nach einem neuen Absoluten zu finden. Arendt ging es um die spezifische Rationalität des Politischen unter Bedingungen der Moderne, d.h. um die Möglichkeit, die Entscheidungen, die im Bereich des Politischen gefällt werden, nicht in die Autonomie des Individuums zu stellen, sondern nach interpersonal gültigen Maßstäben zu beurteilen ${ }^{125}$. Freiheit im Sinne Arendts setzt voraus, dass die Menschen jenseits von individueller Dezision auf der einen, sowie der objektiven Rationalität von Sachzwängen auf der anderen Seite, aus unterschiedlicher Perspektive zu gemeinsamen Urteilen gelangen können. An die Stelle des Rationalitätsprinzips der Einheit mit sich selbst, der Identität, soll ein Vernunftprinzip der Pluralität treten ${ }^{126}$.

Den Anknüpfungspunkt hierzu findet Arendt in Kants Unterscheidung zwischen bestimmender und reflektierender Urteilskraft ${ }^{127}$. Anders als die bestimmende Ur-

121 Max Weber, GPS > aaO. (FN 35), S. 560.

122 So etwa Wilhelm Hennis, aaO. (FN 7), S. $229 f$.

123 Max Weber, GPS, aaO. (FN 35), S. 559.

124 So etwa in Hannah Arendt, Elemente und Ursprünge totaler Herrschaft, München 1986, S. 35, Arendt on Arendt, aaO. (FN 80), S. 336.

125 In diesem Sinn hat vor allem Ernst Vollrath das Werk Hannah Arendts interpretiert und daran anknüpfend seine "philosophische Theorie des Politischen « entfaltet. Vgl. dazu insbesondere Ernst Vollrath, Grundlegung einer philosophischen Theorie des Politischen, Würzburg 1987.

126 Vgl. ebd., S. 20f.

127 Vgl. Imanuel Kant, Kritik der Urteilskraft, hrsg. v. Karl Vorländer, Hamburg 1990, Einleitung, S. 15. 
teilskraft, die nach Regeln und Gesetzen verfährt, das Besondere unter das Allgemeine subsumiert und zu zwingenden Schlüssen gelangt, muss die reflektierende Urteilskraft, die nach Kant unseren Geschmacksurteilen zugrunde liegt, ohne feste Maßstäbe und Regeln auskommen. Sie kann sich nicht auf logisch zwingende Verstandesoperationen gründen, sondern muss durch das Angeben von Gründen um Zustimmung werben. Dazu bedarf es der »erweiterten Denkungsart«. Kant meint damit, wir könnten in Fragen der Ästhetik zu verallgemeinerungsfähigen Urteilen kommen, indem wir von den »subjektiven Privatbedingungen unseres Urteils« abstrahieren und den Gegenstand, um den es geht, aus der Sicht anderer betrachten ${ }^{128}$. Arendt glaubt, hier den Ansatzpunkt für ein politisches Rationalitätskonzept gefunden zu haben, das den Bedingungen säkularisierter und unwiderruflich pluralisierter moderner Gesellschaften entspricht. Wenn wir in der Lage sind, »die Dinge nicht nur aus der eigenen, sondern aus der Perspektive aller anderen, die präsent sind, zu sehen « ${ }^{129}$, wird der Verlust fester, allseits anerkannter Maßstäbe weder zum normativen Nihilismus noch zum »unüberbrückbaren tödlichen Kampf « der Werte ${ }^{130}$ führen.

Allerdings kann das reflexive Urteilen nicht so einfach funktionieren wie die Tätigkeit des Verstandes beim logischen Schließen und Kalkulieren. Es bedarf der Präsenz anderer.

»Was die Präsenz des Selbst für die formale Widerspruchslosigkeit der Gewissensethik ist, ist die Präsenz der anderen für das Urteilen. Ihm kommt daher eine gewisse konkrete Allgemeingültigkeit $\mathrm{zu}$, aber niemals eine universale Gültigkeit überhaupt. Der Anspruch auf Geltung kann nie weiter reichen, als die anderen, an deren Stelle mitgedacht wird « 131 .

Damit liegt eine offensichtliche Schwäche der Hoffnung auf die politische Rationalität des reflexiven Urteils in dessen Voraussetzungen, die nach Arendts eigener kulturkritischer Analyse ja der Siegeszug der modernen Arbeitsgesellschaft und ihres Konformismus untergräbt. Zwar benötigt das reflexive Urteil weder eine verbindliche Tradition noch gemeinsame letzte Werte. Damit es sein Rationalitätspotential entfalten kann, bedarf es jedoch eines pluralen öffentlichen Raumes, einer geteilten Welt, einschließlich gemeinsamer politischer Institutionen sowie subjektiv der Bereitschaft und des Vermögens der Individuen, sich in die Perspektive anderer zu versetzen. Obwohl Arendts Gesellschaftskritik das Schwinden dieser Voraussetzungen diagnostiziert, sieht sie in den Institutionen der amerikanischen Republik und dem Gemeinsinn ihrer Bürger noch Gegenkräfte, die ihr immer wieder Anlass zur Hoffnung geben ${ }^{132}$.

128 Ebd., $\mathbb{} 40$.

129 Hannah Arendt, KP, aaO. (FN 90), S. 299.

130 Max Weber, GAW, aaO. (FN 24), S. 507.

131 Hannah Arendt, KP > aaO. (FN 129), S. 298.

132 Am deutlichsten etwa in ihrer Interpretation der amerikanischen Studentenbewegung (vgl. Hannah Arendt, »Ziviler Ungehorsam《, aaO. (FN 117). Ähnlich aber auch bereits in ihren frühen Eindrücken von amerikanischen Durchschnittsbürgern, die Gemeinsinn zeigten und sich etwa für die Rechte ihrer Mitbürger einsetzten (Hannah Arendt, Hannah Arendt, Karl Jaspers. Briefwechsel 1926-1969, München 1985, S. 66) 
Ob Hannah Arendt mit ihrer Anleihe bei Kants Ästhetik tatsächlich einen modernitätsgerechten Ausweg aus dem Dilemma der Letztbegründung weist, ist in der Literatur grundsätzlich umstritten ${ }^{133}$. Unabhängig davon, wie diese Frage beantwortet wird, liegt der für uns entscheidende Gesichtspunkt in Arendts Bestimmung des Politischen als eines Bereiches, in dem das Handeln Verschiedener eine eigene, beschränkt verallgemeinerbare Vernunft hervorbringt. Den Maßstab für die Rationalität politischen Handelns bildet damit nicht mehr seine $Z$ weckmäßigkeit für willkürlich und vorpolitisch gewählte Werte, sondern die Zustimmung im Urteil der anderen.

\section{Fazit:}

Die dezisionistische Perspektive Webers habe ich weiter vorne als formal, pessimistisch-elitär und, im Gegensatz zu den versachlichten Verhältnissen moderner Gesellschaften, als direkt-herrschaftlich bezeichnet. Die ersten beiden Charakterisierungen treffen auf den ersten Blick auch auf Hannah Arendts Denken zu. Tatsächlich wurde ihr »enthusiastischer Politikbegriff «(Vollrath) wiederholt als formal und elitär kritisiert. Auf Unverständnis stößt insbesondere, dass Arendt das Politische nicht von Inhalten her bestimmt und spezifischer noch, dass sie das Gesellschaftliche als einen Bereich quasi naturhafter Notwendigkeit explizit aus dem Bereich des Politischen ausschließen will ${ }^{134}$.

133 Der Streit geht einmal darum, ob Arendt zu den »anti-foundationalists « zu rechnen sei, oder aber ihr Denken in einem anthropologischen Universalismus gründe, der eine Ethik radikaler Intersubjektivität impliziere. Die erste Position vertritt etwa Margaret Canovan (vgl. Margaret Canovan, Hannah Arendt. A Reinterpretation of Her Political Thinking, Cambridge 1992, S. 191), die zweite etwa Seyla Benhabib (vgl. Seyla Benhabib, The Reluctant Modernism, aaO, (FN 4) 1996, S. 195). Unterstützt man die erste Position, so lässt sich wiederum streiten, ob Arendt damit in gefährliche Nähe zu Nietzsche und der postmodernen Destruktion jeder Moralität gerät, oder aber mit ihrem auf eine gemeinsame, plurale Welt beschränkten Anspruch der Verallgemeinerung nicht gerade einen Ausweg zwischen den Absolutheitsansprüchen eines moralischen Universalismus und den jede Moral in Macht auflösenden Dekonstruktivisten weist. Für den Vorwurf der Amoralität steht vor allem Kateb (George Kateb, Hannab Arendt. Politics, Conscience, Evil. Totowa NJ 1984 und »The Questionable Influence of Arendt (and Strauss)« in: Peter Graf Kielmansegg / Horst Mewes / E. Glaser-Schmidt (eds.) Cambridge/New York 1995), für die Gegenposition prominent Villa (vgl. etwa Dana R. Villa, »Beyond Good and Evil. Arendt, Nietzsche, and the Aesthetization of Political Action « in: Political Theory, Vol. 20, 2/1992 u. ders., Arendt and Heidegger, aaO. (FN 61) sowie Curtis (vgl. Kimberley Curtis, »Aesthetic Foundations of Democratic Politics in the Work of Hannah Arendt « in: C. Calhoun / J. McGowan (eds.), Hannab Arendt and the Meaning of Politics, Minneapolis 1997 und Kimberley Curtis, Our Sense of the Real. Aesthetic Experience and Arendtian Politics, Ithaca/London 1999).

134 Entsprechende Einwände finden sich knapp und verständlich zusammengefasst im Gespräch Arendts mit Richard Bernstein, Hans Jonas, Mary MacCarthy u.a. (vgl. AoA, aaO. $(\mathrm{FN} \mathrm{80)})$. 
Diese Parallele hat einen Grund darin, dass Weber wie Arendt versuchen, das Politische durch einen Handlungstyp zu bestimmen, der gegen die Verselbständigung formaler Rationalität hier, bzw. das »automatische Funktionieren « moderner Arbeitsgesellschaften dort, Sinn stiften und Freiheit ermöglichen soll. Für beide gilt auch, dass der jeweilige Handlungstyp, bei Weber die willkürliche Entscheidung des Individuums für letzte Werte und deren zweckrationale Verwirklichung, bei Arendt das Handeln vor und mit anderen, Selbstzweck ist. Dies setzt beide dem Vorwurf der Amoralität aus, was insoweit auch plausibel scheint, als sie in der Tat die Bindung der Politik an allgemeingültige letzte Werte ablehnen, bzw. unter modernen Bedingungen für unmöglich halten. Im Gegensatz zu den Vertretern der Frankfurter Schule haben sich Weber wie Arendt von jeder, auch von einer melancholischen Orientierung auf eine »objektive Vernunft « (Horkheimer) gelöst. Während jedoch Webers Dezisionismus die Gefahr birgt, mit der Lösung von allgemeingültigen letzten Werten Zweckrationalität und Herrschaft normativ zu entgrenzen und zu radikalisieren, kann Arendt mit ihrer Bestimmung des Politischen als Sprechen und Handeln vor und mit anderen nicht nur eine fundamentale Kritik der herrschaftskategorialen Wahrnehmung des Politischen leisten, sondern darüber hinaus eine Perspektive seiner immanenten Rationalisierung und normativen Selbstbeschränkung weisen.

Erstaunlicherweise geht dieser Gegensatz einher mit einer positiven Würdigung der amerikanischen Demokratie, durch die sich beide Autoren deutlich von anderen kulturkritischen Denkern des 20. Jahrhunderts unterscheiden. Bei Weber wie Arendt lässt sich von einer Demokratisierung der Kulturkritik sprechen, und bei beiden kommt dabei der jeweiligen Interpretation der amerikanischen Demokratie eine Schlüsselrolle zu.

Weber sieht in den zeitgenössischen Entwicklungen zur plebiszitären Führerdemokratie die Möglichkeit, die wertrationalen Entscheidungen der politischen Führer herrschaftlich durchzusetzen. Für ihn bildet dies das entscheidende Gegengewicht zur rationalisierten Fremdbestimmung des modernen Menschen durch Markt und Bürokratie.

Arendt geht zurück zur amerikanischen Revolution und sieht dort im freien Handeln unter Gleichen, in den Formen politischer Selbstorganisation und Partizipation das neuzeitliche Gegenmodell zum Funktionalismus der Arbeitsgesellschaft.

In beiden Fällen handelt es sich um deutsche Lesarten der amerikanischen Politik: Webers herrschaftskategoriale, in der Tradition des deutschen Staatsrechts stehende Wahrnehmung des politischen Kampfes in der Demokratie kann das bürgerschaftliche oder zivilgesellschaftliche Element in den angelsächsischen Ländern nur als unzeitgemäßes Relikt wahrnehmen. Demokratie wird zur Auslese von dezisions- und herrschaftsfähigen Führern.

Arendt politisiert die existentialistische Kulturkritik, indem sie die bürgerschaftlichen Formen der amerikanischen Republik als Überwindung der bis zu Platon zurückreichenden Tradition interpretiert, Politik in den Kategorien von Subjekt und Objekt, von Zweck und Mittel und damit herrschaftszentriert zu denken. Herrschaft und Gewalt kann sie so als Abweichung von den revolutionären Ursprüngen 
neuzeitlicher Politik normativ kritisieren, ohne auf eine wie immer geartete geschichtsphilosophische Verkörperung der Vernunft zurückgreifen zu müssen.

In Bezug auf beide, Weber wie Arendt, stellt sich allerdings die grundsätzlichere Frage, ob wir ihr kulturkritisches Ausgangsproblem, nämlich die zunehmende Erosion von Sinn und Handlungsmöglichkeiten durch die funktionale Organisation moderner Gesellschaften, überhaupt noch teilen.

\section{Zusammenfassung}

Max Weber wie Hannah Arendt sind stark beeinflusst von der deutschen Tradition der Kulturkritik. Im Gegensatz zur Frankfurter Schule sehen jedoch beide in demokratischer Politik die bedeutendste Gegenkraft zum kritisierten Sinn- und Freiheitsverlust in modernen Gesellschaften. Während jedoch Max Weber in der sich damals in den Vereinigten Staaten abzeichnenden plebiszitären Führerdemokratie einen letzten Hort von Wertrationalität und freien Entscheidung sieht, interpretiert Arendt die amerikanische Revolution des 18. Jahrhunderts als neuzeitliche Verwirklichung politischer Freiheit. Der Beitrag zeigt, dass die Modernitätskritik von Arendt und Weber, trotz auffallender Parallelen, auf grundverschiedenen theoretischen Konzepten basiert. Er argumentiert, dass die vermeintlich apolitischen, existenzialphilosophischen Begriffe der Kulturkritik Arendts es ihr ermöglichen, politische Freiheit unter den Bedingungen moderner Gesellschaften zu denken, während Webers sozialwissenschaftliche Theorie der Rationalisierung zu politischem Dezisionismus und einem herrschaftlichen Politikbegriff führt.

\section{Abstract}

Both, Max Weber as well as Hannah Arendt are strongly influenced by the German tradition of cultural criticism. And both, unlike the first generation of the Frankfurt School, see democratic politics as the most important counterforce to the loss of meaning and to the loss of freedom which they denounce in modern societies. However, whereas Max Weber understands the emerging plebiscitary leadership democracy in the United States of his time as a (last) resort of value rationality and free decision, Hannah Arendt interprets the American Republic of the $18^{\text {th }}$ century and its institutions as a modern realm of acting with others, i.e. of political freedom. The article shows that Arendt's and Weber's criticism of modernity are, in spite of striking parallels, based on very different theoretical concepts. It argues that Arendt's presumably a-political categories of Existenz-philosophy open up a perception of political freedom under the conditions of modernity, whereas Weber's scientific concept of rationality leads to political decisionism and a despotic concept of politics. 